\title{
A Critical Review of Proactive Detection of Driver Stress Levels Based on Multimodal Measurements
}

\author{
MOHAMMAD NAIM RASTGOO, BAHAREH NAKISA, ANDRY RAKOTONIRAINY, \\ VINOD CHANDRAN, and DIAN TJONDRONEGORO, Queensland University of Technology
}

\begin{abstract}
Stress is a major concern in daily life, as it imposes significant and growing health and economic costs on society every year. Stress and driving are a dangerous combination and can lead to life-threatening situations, evidenced by the large number of road traffic crashes that occur every year due to driver stress. In addition, the rate of general health issues caused by work-related chronic stress in drivers who work in public and private transport is greater than in many other occupational groups. An in-vehicle warning system for driver stress levels is needed to continuously predict dangerous driving situations and proactively alert drivers to ensure safe and comfortable driving. As a result of the recent developments in ambient intelligence, such as sensing technologies, pervasive devices, context recognition, and communications, driver stress can be automatically detected using multimodal measurements. This critical review investigates the state of the art of techniques and achievements for automatic driver stress level detection based on multimodal sensors and data. In this work, the most widely used data followed by frequent and highly performed selected features to detect driver stress levels are analyzed and presented. This review also discusses key methodological issues and gaps that hinder the implementation of driver stress detection systems and offers insights into future research directions.
\end{abstract}

CCS-Concepts: Human-centered computing $\rightarrow$ Ubiquitous and mobile computing; General and reference $\rightarrow$ Surveys and overviews; Computing methodologies $\rightarrow$ Machine learning algorithms

Additional Key Words and Phrases: Driver stress level detection, multimodality, physiological signals, ECG, EDA, respiration, physical signals, vehicle dynamic data, contextual data, machine learning, real-time stress recognition system

This work is supported by a QUT Postgraduate Research Award (QUTPRA).

Authors' addresses: M. N. Rastgoo and B. Nakisa, School of Electrical Engineering and Computer Science, Faculty of Science and Engineering, Queensland University of Technology, 2 George St., Brisbane QLD 4000; emails: mohammadnaim. rastgoo@qut.edu.au, Bahareh.nakisa@qut.edu.au; A. Rakotonirainy, School of Psychology and Counselling, Research CARRSQ, Faculty of Health, Queensland University of Technology, Victoria Park Rd., Kelvin Grove QLD 4059; email: r.andry@qut.edu.au; V. Chandran, School of Electrical Engineering and Computer Science, Faculty of Science and Engineering, Queensland University of Technology, 2 George St., Brisbane QLD 4000; email: v.chandran@qut.edu.au; D. Tjondronegoro, School of Business and Tourism, Southern Cross University, Bilinga, QLD, Australia 4225; email: dian.tjondronegoro@scu.edu.au.

Permission to make digital or hard copies of all or part of this work for personal or classroom use is granted without fee provided that copies are not made or distributed for profit or commercial advantage and that copies bear this notice and the full citation on the first page. Copyrights for components of this work owned by others than ACM must be honored. Abstracting with credit is permitted. To copy otherwise, or republish, to post on servers or to redistribute to lists, requires prior specific permission and/or a fee. Request permissions from permissions@acm.org.

(C) 2018 Association for Computing Machinery.

0360-0300/2018/09-ART88 \$15.00

https://doi.org/10.1145/3186585 


\section{ACM Reference format:}

Mohammad Naim Rastgoo, Bahareh Nakisa, Andry Rakotonirainy, Vinod Chandran, and Dian Tjondronegoro. 2018. A Critical Review of Proactive Detection of Driver Stress Levels Based on Multimodal Measurements. ACM Comput. Surv. 51, 5, Article 88 (September 2018), 35 pages.

https://doi.org/10.1145/3186585

\section{INTRODUCTION}

Driving is a perceptual motor skill that entails multiple situations and consequently results in varying levels of stress. Stress is an important factor in driving because it can increase the risk of getting into a vehicle crash by affecting driver performance. The world death records issued by the World Health Organization (WHO) show that road traffic crashes cause over 1.2 million deaths annually (Sauerzapf 2012). This issue creates a significant cost in terms of loss of life and productivity for governments and societies (Blincoe et al. 2015). Stress has been identified as one of the most significant reasons for vehicle crashes (Katsis et al. 2015). Australian national crash reports also show that stress is among the 10 leading causes of fatal crashes (Beanland et al. 2013). Aside from increasing the risk of crashing, stress can also affect the general health of professional drivers. Professional drivers are those whose occupation is driving vehicles, such as bus, taxi, and heavy transport drivers. This group of drivers is exposed to continuous stressors in their everyday work. Findings from a European survey on working conditions in 2005 show that workers in the transport sector experience more stress than the average worker population (European Working Conditions Survey 2005). In this research, about 32\% of drivers who worked in "land transport" reported that work stress affects their health (Copsey et al. 2010), confirming earlier findings that driving is one of the unhealthiest occupations (Evans and Carrère 1991; Hanzlíková 2005).

Motivated by the need to address the significant costs of driver stress, this article investigates the state-of-the-art achievements and existing challenges in building a practical system that can continuously, unobtrusively, and automatically detect driver stress levels at an early stage. A shortterm benefit arising from such a system would be to increase driver safety by predicting dangerous driving situations and alerting the driver proactively. In addition, a long-term benefit would be prevention of harmful, long-lasting consequences of stress in professional drivers. Thanks to recent developments in ambient intelligence, such as sensing technologies, pervasive devices, context recognition, and communications, driver stress levels can be automatically detected using a combination of multimodal measurements.

This article focuses on reviewing the modalities used to measure driver stress levels by reviewing literature published between 1990 and 2017. Based on a proposed framework for a multimodal driver stress recognition system, it also reviews state-of-the-art techniques for automatic driver stress recognition and investigates their efficiencies. Based on the reviews, this article discusses the existing methodological challenges and recommends ideas for future work in building a practical system that can continuously, unobtrusively, and automatically detect driver stress levels. To the best of our knowledge, this is the first review that investigates the data, techniques, and issues that need to be addressed for implementing such a system in vehicles to increase driver safety. For surveys on stress recognition and classification, we refer the reader to Sharma et al. (2012). Our work is also different from a previous review that focuses on recognition of office work-related stress through multimodal data (Alberdi et al. 2016).

This review is structured as follows: Section 2 defines stress-related issues and describes driver stress consequences in detail. Section 3 introduces the multimodal nature of driver stress and discusses the stress analysis methods for each modality. Section 4 discuses different pattern-matching techniques used to build multimodal stress detection models. Section 5 discusses computational 
methods that have been employed in analyzing driver stress. Section 6 defines a framework of a proactive detection system of driver stress levels and discusses issues related to such a system. Finally, Section 7 presents our main conclusions and discusses open problems and ideas for future work.

\section{STRESS AND CONSEQUENCES FOR DRIVING}

Stress can be defined as a nonspecific bodily response to a combination of external demands and internal concerns. Stress can be distinguished in the concept of eustress (positive stress) and distress (negative stress) (Selye 1974). Eustress occurs when an individual's perceived ability to cope outweighs his or her perceived external demands. It can help an individual to increase his or her creativity and productivity and motivate him or her to perform a specific task. However, distress occurs when there is an imbalance between an individual's ability to cope and his or her perceived external demands. This sort of stress is dangerous and can cause negative consequences. In daily life, we often use the term "stress" to describe negative stress rather than positive stress. In this review, the term "stress" also refers only to negative stress.

Stress is generally caused by an unpleasant intervener in the form of real events, called a stressor, and is affected by the subject's individual and ambient parameters, which are called the subject's context. Therefore, the external demands, known as a stressful situation, refer to the stressor and ambient parameters, while the internal concerns refer to the subject's individual parameters. There are three classes of stress: acute, episodic, and chronic. Acute stress is caused by a short-term stressor, and cortisol is secreted at high levels during the initial phase of stress to prepare the body for a fast and appropriate reaction (Selye 1956). This type of stress ends quickly, and the body soon reverts to its balance point. In a stressful life/work environment, acute stressful situations may occur from time to time, causing another stress type, called episodic stress. In addition, a stressor that lasts for a long time may cause chronic stress, which is harmful to human health.

Fast-changing driving conditions can cause short-term stressors; therefore, drivers in these situations are more likely to be exposed to acute and episodic stress. Short-term stressors related to driving tasks can be classified into five categories: (1) social interactions, (2) unexpected situations, (3) other drivers' or pedestrians' behaviors, (4) events that impact time schedule, and (5) difficult driving due to urban planning (Rodrigues et al. 2015). Ambient factors, such as road conditions or traffic density, and internal factors, such as a driver's low experience or high mental workload, can also affect stressful driving situations.

Professional drivers are prone to prolonged or continuous stressors, which can lead to chronic stress. These stressors are generally caused by the driver's environment, job-related tasks, and organizational issues (John et al. 2006). Environmental stressors may include the ergonomic design of a vehicle's cabin, ambient noise, weather conditions, road rage, and traffic congestion (John et al. 2006). Job-related task stressors may include security responsibilities, following a specific working schedule, and prolonged social isolation (Rodrigues et al. 2015). Organizational issue stressors may include limits on a driver's decision-making authority (John et al. 2006).

\subsection{Stress and Vehicle Crash}

Successful driver performance is a fundamental issue for preventing vehicle crashes, and a driver's success is often determined by adapting his or her behavior with respect to changes in environment and complexity of context while driving (Schmidt and Lee 2005). Unsuccessful driver performance causes over $80 \%$ of vehicle crashes that result in fatalities and injuries (Stanton and Salmon 2009). Driver performance can be negatively affected by stress, which then can cause traffic violations and crashes (Beirness 1993; Simon and Corbett 1996). This is because stress causes both high mental workload (Wiberg et al. 2015) and negative moods in drivers (Frasson et al. 2014). 
High mental workload may result in inadequate information processing and imperfect perception (Lenné et al. 1997; Boyle et al. 2008). It can also cause cognitive distraction, which is one of the most common reasons for drivers' distraction. A driver's cognitive distraction may lead to impaired decision-making capabilities (Baddeley 1972). As a result, high mental workload due to stress leads to deterioration of driver performance (Wiberg et al. 2015).

Negative moods, such as anger and aggressiveness, can be induced in stressful driving situations (Allen et al. 1993; Frasson et al. 2014; Lundberg et al. 1994). Driving in an angry mood can increase risky patterns of driving, such as high speed, switching lanes, and running red lights (Allen et al. 1993). Such aggressive behaviors are dangerous, and associated with higher probabilities of road traffic crashes (Parry 1968; Deffenbacher et al. 2003).

\subsection{Chronic Stress Consequences for Professional Drivers}

Chronic stress is a serious issue for professional drivers, as it adversely impacts on their general physical and mental health. Bus drivers' exposure to chronic occupational stress can increase their chance of developing cardiovascular problems (Baevskii et al. 2009). Gastrointestinal and musculoskeletal disorders are also considered a consequence of chronic stress for professional drivers (Hanzlíková 2005). Posttraumatic stress disorder is also reported as a common chronic mental health issue in ambulance and bus drivers (Boyer and Brunet 1996; Berger et al. 2007).

\section{CURRENT METHODS TO MEASURE STRESS MODALITIES}

The human body generally employs the fight-or-flight response to instantaneously make decisions during a stressful situation, including how to avoid its negative outcomes-for example, avoiding accidents during dangerous driving conditions. The nervous system is responsible for executing this strategy in three consecutive steps: sensing, perceiving, and responding to stress (Aydede and Robbins 2009). Central and peripheral nervous systems are the two key components that control different organs of the body and work together to regulate the fight-or-flight response (Monroe 2009). In this regard, the sensory nerves collect information related to a stressful situation (stressor and ambient parameters) and send it to the brain as the main core of the central nervous system (sensing). The brain processes the received information and individual parameters to perceive the stress level (perceiving), and finally sends proper responses through somatic and autonomic motor nerves to different parts of the body for voluntary and involuntary reactions (responding).

The fight-or-flight response can be monitored through different modalities to measure driver stress. These modalities include driving stressors, the driver's ambient and individual parameters (contextual data), and the driver's psychological, physiological, and physical responses to stress. The driver's psychological responses, being related to the driver's mental activity (Rothkrantz et al. 2004), reflect the stress level perceived by the brain in a stressful driving situation. Physiological responses are the result of whole body function (Baum 1997). The driver's nonobservable responses are regulated through the autonomic nervous system (ANS). The driver's physical responses are voluntary and observable behaviors (Horlings et al. 2008) that the brain regulates through the somatic nervous system. In the rest of the section, the information associated with these modalities in the domain of driver stress recognition is reviewed.

\subsection{Psychological Evaluation}

Psychological evaluation is mostly used as a stress marker or ground truth in stress recognition domains. There are various questionnaires, known as clinical stress questionnaires, which are employed for this evaluation, such as the Stress Self Rating Scale (Alberdi et al. 2016). A weakness of this approach is that drivers may find it difficult to recall acute stressful events, as the questionnaires are usually conducted at the end of experiments (Singh and Queyam 2013a). There are also 
some self-reporting methods that can provide flexible ways to measure driver stress, either during driving (Rodrigues et al. 2015) or after driving (Healey and Picard 2005). A common method is the $\mathrm{N}$-point Likert scale: drivers score their stress level using a predefined range of scores, such as 1 (no stress) to 6 (high stress). Another method is categorization: drivers select a category of stress from a predefined stress category group, such as low, medium, and high stress. However, it should be noted that filling out questionnaires or self-reporting during driving is not practical for safety reasons.

Annotation is a common technique that helps drivers to answer abstract self-reporting methods. This technique can be divided into offline, online, and combined annotation, based on when drivers carry out the psychological evaluation. In the offline annotation, drivers annotate their perceived stress levels for different driving events that were recorded during their driving. Some studies have recorded the driving events using a camera or GPS tracker to help drivers in recalling their stress levels (Healey and Picard 2005; Rigas et al. 2011). In the online annotation, the driver annotates his or her perceived stress levels for different driving events while he or she is driving, rather than recalling them afterward. One online annotation technique asked drivers to record their mental strain, on a scale of 1 to 10, every 10 minutes (Eilebrecht et al. 2012). However, this study noted that increasing the frequency of annotation will increase the driver's mental workload. Using a combined method to incorporate both online and offline annotation, a study by Rodrigues et al. (2015) asked bus drivers to wear smart clothes, called a vital jacket, and to tag stressful situations by pressing an embedded button on the jacket while driving. They also added a stress thermometer system to confirm the driver's stress level while driving, on a scale of not-at-all stressful to extremely stressful. The driving paths were recorded using GPS during the experiment and then visualized using Google Earth. The bus drivers, by reviewing the recorded paths, provided a brief explanation for the stressful events followed by their stress intensity evaluation.

\subsection{Measuring Drivers' Internal Physiological Responses}

The autonomic nervous system regulates physiological stress responses through the sympathetic nervous system (SNS) and the parasympathetic nervous system (PNS). When the human body needs to react quickly to eliminate a stressor, the SNS is activated. The PNS is activated when the body needs to be in a relaxed state. These physiological stress responses can be measured through different biosignals (biochemical or physiological). The level of adrenaline and cortisol in various body fluids such as blood, saliva, or urine can be used as the two main biochemical measurements to detect stress (Wijsman 2014), as stressful situations increase the level of these hormones in the body. Although adrenaline is a well-known biomarker for stress, measuring the level of this hormone to detect driver stress is not recommended because it is measured by invasive methods, such as blood sampling. In contrast, cortisol levels can be determined from saliva, which does not require invasive sampling methods. Other biosignals used to detect driver stress levels are physiological data such as heart rate activity, blood pressure, electrodermal activity, respiration response, muscle activation, skin temperature, and pupillary response.

3.2.1 Cortisol Level. The sympathetic nervous activity caused by driver stress can be evaluated using salivary cortisol levels (Deguchi et al. 2006). In a study, salivary amylase activity was employed to assess acute stress effects on vehicle drivers (Yamaguchi et al. 2006). In this study, 20 young female drivers were recruited, and each driver spent 21 minutes in a driving simulator. During this time the participant's salivary amylase activity was measured every 3 minutes. This study demonstrates two points: first, the feasibility of measuring this biomarker noninvasively during driving tasks, to facilitate monitoring of driver stress levels; second, the use of saliva as a biomarker to obtain an objective ground truth for assessing driver stress, as an alternative to 
the current subjective ground-truth methods. This method is able to measure driver stress more accurately while driving.

Measuring the cortisol hormone to evaluate stress for a long time has some limitations because this hormone is largely influenced by the time of day rather than stress. It has been shown that a person's cortisol level is at its peak when the person is awake and decreases gradually during the day (Chennaoui et al. 2016). Therefore, the time of day when sampling is done must be controlled when assessing cortisol level.

3.2.2 Heart Activity. Driving in stressful situations activates the sympathetic part of the ANS, which results in a sudden increase of heart activity and puts the driver's body in the fight-or-flight response mode against the stressor (Soman et al. 2013). This means that the heart pumps blood through the body faster and so oxygen is delivered faster to the body's organs to prepare the driver to react. Among different indicators of heart rate activity, heart rate and heart rate variability have been widely used in detection of driver stress levels. Generally, these two parameters can show the fluctuation of the ANS, which directly affects heart activity (Camm et al. 1996), allowing the physiological body response toward a stressful situation to be observed. Heart rate (HR) is defined as the number of times the heart beats per minute, while heart rate variability (HRV) is defined as the time fluctuations between sequences of successive heart beats. These parameters normally can be extracted from electrocardiogram (Sörnmo and Laguna 2006) or photoplethysmogram (Elgendi 2012) signals. Electrocardiogram (ECG or EKG) signals present the heart's electrical activity as it fluctuates within time (Price 2010), while photoplethysmogram (PPG) signals are an indicator of the blood flow rate that is controlled by the heart's pumping action (Sahni 2012).

During stressful driving situations, HR and HRV signal patterns change due to the fluctuations in ANS activities, which makes them good indicators of a driver's stress (Munla et al. 2015). Stress is directly related to HR, as stress increases the driver's heart rate. Mean and standard deviation of HR are two common statistical features used in detecting driver stress levels. HRV signals are often analyzed in time and frequency domains to detect driver stress levels. In the time domain, HRV signals are indirectly related to driver stress (Eilebrecht et al. 2012; Miller and Boyle 2013). Mean normal-to-normal intervals (MNN), standard deviation of normal-to-normal intervals (SDNN), square root of the mean squared difference of successive normal-to-normal intervals (RMSSD), and number of pairs of successive normal-to-normal intervals that differ by more than 50ms (PNN50) have been used by several studies as HRV time-domain features. (See Table 1.) The HRV time-domain features are influenced largely by momentary ANS activities; thus, HRV timedomain analysis can be used to measure instantaneous driver stress responses (Lee et al. 2007). In the frequency domain, high-frequency (HF) and low-frequency (LF) ranges between 0.2 and $0.4 \mathrm{~Hz}$, and 0.05 and $0.2 \mathrm{~Hz}$, respectively, are the most common power spectrum parameters used for detecting driver stress levels. The ratio of power in these two frequency bands (LF/HF) can be considered an indicator of sympathovagal balance (Montano et al. 1994), which has a direct correlation with a driver's stress and mental workload level (Healey and Picard 2005; Miller and Boyle 2013; Heikoop et al. 2017). As a driver's stress and mental workload levels increase, HF values decrease while LF values increase. In addition to HR and HRV signals, a driver's beat-by-beat mean blood pressure and normalized blood volume pulse have been shown to have a correlation with the driver's stress level (Yamakoshi et al. 2008). Total peripheral resistance is also shown as a cardiovascular feature related to driver stress (Yamakoshi et al. 2008).

In order to classify drivers' stress levels, several data analysis methods have been used by researchers. Wang et al. (2013) monitored HRV signals to classify drivers' stress into two levels using a K-Nearest Neighbors (KNN) classifier and achieved an average recognition rate of $97.78 \%$ via fivefold cross-validation. The HRV signal was divided into 5-minute intervals with $50 \%$ overlap and 
each interval was mapped to 56 features. In another study, several features were extracted from physiological, physical, and contextual modalities and used to detect drivers' stress (Rigas et al. 2011). Electrocardiogram signals, electrodermal activity, and respiration signals were collected as physiological responses in the study, among which ECG features were shown to have the highest correlation to drivers' stress. The proposed model achieved $86 \%$ detection accuracy. Singh et al. (2013) applied different artificial neural network architectures to classify drivers' stress into three levels. In the study, 39 features from different physiological signals (HRV, electrodermal activity, respiration rate, and PPG) were extracted and then a subset of the features was selected using a feature selection technique. The proposed model using a layer-recurrent neural network classifier was found to be the optimal model, with a precision of $89.23 \%$.

The features extracted from drivers' HR and HRV signals have been shown to be affected in different ways by contextual parameters such as vehicle maneuvering, traffic volume, alerting events, road direction (clockwise or counterclockwise), driving across various routes, driving tasks, and drivers' emotions and fatigue. The findings show changes in drivers' HR signals during some driving tasks (Hartley et al. 1994; Liu et al. 2004). Some studies also show that HR value has an indirect relationship to driving time and a direct relationship to vehicle maneuvering, traffic volume, and road direction (Simonson et al. 1968; Platt 1970). Compared with HR, HRV signals (both in time and frequency domains) retain their characteristics related to driver stress regardless of the direction of travel (Miller and Boyle 2013). However, HRV is shown to increase with driving time and decrease after alerting events. Regarding drivers' individual factors, both HRV and HR can react to negative and positive driver emotions and also be affected by driver fatigue. Therefore, HR and HRV responses toward stressful situations cannot be the same under different driving conditions.

3.2.3 Electrodermal Activity. Another reliable physiological signal to detect driver stress is electrodermal activity (EDA), also called skin conductance response (SCR) or galvanic skin response (GSR). A body's physiological response to a stressful situation results in continuous variations in the electrical activity of skin, which can be measured on the hand (palm area, first and middle fingers) or on the foot (soles) by using EDA sensors. The correlation between EDA signal and drivers' stress levels has been investigated by several studies (Deng et al. 2013; Singh and Queyam 2013a). There are different statistical EDA features that have been commonly used to analyze driver stress, such as mean, standard deviation, mean amplitude, peak rise time, peak amplitude, first absolute difference, and mean of the first difference (see Table 1). EDA response is characterized by a tonic component and short-term phasic responses (Braithwaite et al. 2013). Tonic component or skin conductance level (SCL) can reflect psycho-physical activation and varies between individuals (Kappeler-Setz et al. 2013). The frequency range of this component is between 0 and $0.05 \mathrm{~Hz}$. The average and variance level of the tonic component are two commonly used features in detecting driver stress levels. Compared with SCL as an indicator of psycho-physical activation, the phasic component or SCR can show the body's response to environmental stimuli, such as stressful driving events. The frequency range of this component is between 0.05 and $1.5 \mathrm{~Hz}$. In order to detect driver stress levels, different features such as latency of first SCR, average phasic activity, variance of phasic signal, maximum phasic amplitude, amplitude-sum of SCRs, and phasic area under curve have been extracted from SCR signals (Lanatà et al. 2015). (See Table 1.) Summary of the startle magnitudes, duration and the area of SCR orienting responses, sum of durations, sum of magnitudes, sum of estimated areas, and frequency of occurrence are extracted from EDA features and used to discriminate the drivers' stress levels as well (Healey and Picard 2000).

There are several studies that have used EDA signals with other modalities to enhance the performance of driver stress classification. In one of these studies, 12 features were extracted from the physiological signals of 10 drivers, which include EDA, ECG, and respiration (Healey and 
Picard 2000). The best subset of the features was picked using a sequential forward floating selection (SFFS) algorithm and then the selected features were fed to a KNN classifier to classify four stress levels. The model's accuracy was $86 \%$. Healey and Picard (2005) used EDA, ECG, respiration, and electromyography (trapezius muscle) signals from three drivers to extract 22 features. Then, a linear discriminant analysis (LDA) classifier was used to classify three levels (low, medium, and high) of the drivers' stress. They achieved $100 \%, 97.4 \%$, and $94.7 \%$ classification accuracy for low, high, and medium stress, respectively. When different physiological and physical responses are used to detect three levels of drivers' stress, a study (Lanatà et al. 2015) shows that the extracted EDA features alone have a promising ability to classify the stress levels. All the above-mentioned studies point out that EDA has a high correlation with drivers' stress levels.

Although SCR response is one of the reliable indicators of driver stress, intersubject variability between drivers can lead to fluctuations in the values of SCR features (Urbano et al. 2017). These fluctuations could be the difference in the number of sweat glands on drivers' palms or the difference in electrode positioning while drivers gripped the steering wheel (Healey and Picard 2005). Combining EDA signals and other indicators of driver stress such as HR or HRV can make a better model to cope with this issue (Rigas et al. 2008; Rigas et al. 2012).

3.2.4 Respiration Activity. Change in respiration activity is a physiological response to stress and emotional state. The respiration system serves as a metabolic and homeostatic regulator of depth and speed of breathing (Katsis et al. 2008). Stressors can increase respiration activity. The respiration responses of drivers can be collected in different ways, including wearing belt sensors, placing a thermistor into the nose and mouth, and using a flow meter (Wijsman 2014).

A group of statistical features-such as mean, standard variation, skewness, and kurtosis-is extracted from respiratory signals to detect drivers' stress levels (Lanatà et al. 2015). The result shows that there is a significant correlation between these features and stress levels. Another common feature in this domain is respiration rate (RSPR). This feature is the number of detected peaks in the respiratory signal per minute where each of the peaks is related to a chest expansion (Soman et al. 2013). A high correlation between this feature and drivers' stress levels are reported by Singh et al. (2013). In another study, RSPR was also extracted from 10 drivers' respiratory signals and then divided into high and low breathing rates (Soman et al. 2013). This study also reports a direct relation between RSPR and stress condition. In the case of frequency domain features, spectral power is shown as a good indicator of driver stress level detection (Deng et al. 2012).

The main limitation of using respiration signals for detecting drivers' stress levels is the intersubject variability between drivers' respiration responses. Drivers' respiration responses to the same stressor can vary based on the size of their chest cavity (Healey and Picard 2005).

3.2.5 Muscle Activation Electromyography. Muscular reactions caused by stressful driving situations can occur unconsciously even when no physical activity is required (Healey et al. 1999). Stressful situations can affect this reaction by increasing muscle activity (Lundberg et al. 1994), which can be measured using electromyography (EMG) signals. These signals are recorded from the drivers' shoulders (e.g., the trapezius muscle) or facial muscles (e.g., the masseter muscle) to analyze their stress levels (Healey and Picard 2005; Katsis et al. 2008; Katsis et al. 2011; Zheng et al. 2015).

Statistical features such as mean, root-mean square (RMS), zero crossing rate (ZCR), and rootmean quad (RMQ) are extracted from the EMG signals to detect drivers' stress levels (Katsis et al. 2008; Soman et al. 2014; Zheng et al. 2015). (See Table 1.) Among these features, ZCR has been found to be more correlated to drivers' stress levels than other features, since high levels of driver stress can increase muscle constriction, resulting in a high ZCR value (Soman et al. 2014). 
There are several studies that focused on the fusion of EMG with other physiological signals to improve the system of detecting drivers' stress levels. It has been shown that fusion of ECG with EMG signals to detect two levels of drivers' stress (low and high) is promising (Soman et al. 2014). This fusion achieved $100 \%$ accuracy using a support vector machine (SVM) classifier. Katsis et al. (2011) used several biosensors to collect racing drivers' ECG, EDA, respiratory, and facial EMG signals to classify four emotional states (low stress, euphoria, disappointment, and high stress). In the study, two statistical features, mean and RMS, from 16 facial EMG channels, along with other extracted features from ECG, EDA, and respiratory signals, were used to build two models. The first model classified three emotional states-low stress, high stress, and valence (euphoria, dysphoria)-and achieved $80.8 \%$ accuracy. The second model classified four emotional states-low stress, high stress, euphoria, and disappointment-and achieved $71.9 \%$ accuracy.

3.2.6 Skin Temperature. Another physiological signal used to detect driver stress is skin temperature (ST). In stressful driving situations, SNS activity causes peripheral vasoconstriction in the body, thereby decreasing the driver's skin temperature (Yamakoshi et al. 2007). Current methods can provide a contactless and passive solution for monitoring driver skin temperature continuously by using thermography sensors in the vehicle.

Nose, cheek, forehead, and jaw regions on the face, which contain abundant arterioles and arteriovenous anastomoses, are used as skin temperature features to monitor drivers' stress and mental workload (Yamakoshi et al. 2008). Yamakoshi et al. (2008) measured drivers' skin temperature, cardiovascular response, and contextual parameters to stressful driving situations. Temperature data through the finger (Tsf), nose (Tsn), cheek (Tsch), jaw (Tsj), and forehead regions (Tsfh) are collected. Two cardiovascular parameters, normalized pulse volume (NPV) and total peripheral resistance (TPR), were also used to detect peripheral vasoconstriction. The result shows that a gradual increase in a driver's stress level can cause peripheral vasoconstriction and decrease Tsn and Tsf values as two peripheral components of the face. Due to individual differences between the drivers, Tsf values varied more than Tsn; therefore, Tsn is offered as a better indicator of drivers' stress. Tsn was shown to be influenced by some contextual parameters, such as changes of ambient temperature, radiation temperature, relative humidity, and metabolic changes. Tsch, Tsfh, and Tsj, as three truncal components of the face, were also shown to be entirely dependent on the contextual parameters values rather than drivers' stress. On this basis, the authors used differential temperatures between Tsn and Tsch, Tsfh, and Tsj to eliminate the influence of the contextual parameters from Tsn. (See Table 1.) In another study (Shastri et al. 2008), the thermal signature of drivers' faces in the supraorbital region, which is immediately above the eye socket, was measured to detect the mental workload of drivers. The results show that driving in stressful and distracting situations, including texting and making phone calls, results in increased blood flow to the driver's supraorbital region, increasing the temperature of that region.

3.2.7 Pupillary Dilation. It has been shown that pupillary response is another promising physiological signal for detecting driver stress (Pedrotti et al. 2014). The ANS continuously regulates tuning of pupil size, which is known as pupillary dilation. The pupil is dilated (i.e., its diameter increases) by SNS activity when the body is under stress. To this end, pupil diameter (PD) can be a good indicator of drivers' stress levels.

Pedrotti et al. (2014) employed a classifier based on the neural network and wavelet transform to detect the stress levels of drivers using PD, EDA, and a self-assessment questionnaire. They reduced the sample rate of PD signals from $50 \mathrm{~Hz}$ to $1.5625 \mathrm{~Hz}$, and then applied a Haar wavelet with five decomposition levels to the PD signals to extract meaningful information from low-frequency bands. The result of the study showed that PD could classify drivers' stress levels significantly (79\% accuracy) while the performance of EDA was poor. 
3.2.8 Summary. Among the physiological signals and related features used in the driver stress level detection domain, presented in Sections 3.2.2 to 3.2.7 and in Table 1, several studies showed a strong correlation between ECG, EDA, and respiration signals and driver stress levels. Although the physiological signals are considered reliable indicators for detecting driver stress, obtaining the signals involves obtrusive methods. In real-life driving situations, wearing these sensors continuously may not be practicable.

\subsection{Measuring Drivers' External Physical Responses}

In addition to involuntary physiological responses to stress, a driver may have several external and voluntary reactions known as physical or behavioral responses. Physical responses are used to detect driver distraction ( $\mathrm{Li}$ and Busso 2013; Li et al. 2013), inattention (Bergasa et al. 2008), fatigue (Sahayadhas et al. 2012), mental workload (Marquart et al. 2015), and stress (Fernandez and Picard 2003). The SNS regulates physical responses as conscious and voluntary actions that can be observed directly through the movement of the skeleton, muscles, and tissues of the body, including limbs, fingers, toes, neck, and face, and heard through vocalization. Among these physical responses, data recorded from drivers' faces and speech are used to analyze their stress.

In addition, monitoring dynamic data generated from the vehicle, such as its average speed, is considered a way to indirectly measure drivers' physical responses to stressful driving situations.

3.3.1 Facial Expression. Some studies have focused on analyzing stress levels of drivers using facial expression response (FER) (Rimini-Doering et al. 2001; Paschero et al. 2012). Negative emotions such as anger and disgust, which are caused by driver stress, were recognized by FER (Gao et al. 2014). They used a near-infrared camera to monitor drivers' faces and extracted 49 facial landmarks from each frame of the video using a face tracker. Then, holistic and local texture features from each frame were extracted to recognize drivers' anger and disgust. They achieved $85 \%$ accuracy among 49 subjects using an SVM classifier in a one-versus-all manner.

3.3.2 Vocalization. Drivers' stress (Bořil et al. 2012), behavior (Angkititrakul et al. 2007), distraction (Boril et al. 2010), and mental workload (Chen et al. 2012) may change human vocal production. A voice communication system, which provides an unobtrusive way to track drivers' vocalizations for the purpose of stress analysis, is recommended so that drivers can communicate verbally while maintaining their visual attention on the road (Carter and Graham 2000).

Stress levels of drivers were detected by using their voice waveforms (Fernandez and Picard 2003). Vocalizations of drivers were recorded while they were engaging in several mental tasks such as adding up two numbers. The Teager energy operator and multiresolution analysis via wavelet transforms techniques were used to extract subband energy from each voice waveform frame. Then, cepstral coefficients using the subband energy were calculated for each frame. The classification step, in this study, involved two approaches. In the first approach, the features within vocalizations were used to classify four drivers' stress levels using five different hidden Markov models classifiers. In the second approach, the features across vocalizations were used to classify four drivers' stress levels using SVM and artificial neural network (ANN) classifiers. The highest accuracy obtained among the seven classifiers was $51.22 \%$. Some studies have investigated stress levels of a group of drivers through voice analysis while they were engaging in secondary activities such as doing mental tasks, operating the radio, and talking on a cell phone (Bořil et al. 2012).

3.3.3 Vehicle Dynamic Data. The effects of stress on a driver can be measured indirectly through dynamic data obtained from the vehicle. This data can be collected through different systems such as CAN-Bus (Bořil et al. 2012; Rigas et al. 2012; Rodrigues et al. 2015), GPS tracking 
(Rigas et al. 2012), driving simulators (Cantin et al. 2009; Tango and Botta 2013), mobile phones (Tchankue et al. 2013), and orientation sensors (Lee et al. 2017).

One study used PPG signals and steering-wheel motion data-captured using a 9-degree-offreedom orientation sensor to detect drivers' stress (Lee et al. 2017). In the study, three stress detection models were built using SVM classifiers with different kernel functions. The proposed model using the SVM-RBF kernel function was found to be the optimal, with an accuracy of $95.38 \%$. Lanata et al. (2015) proposed a model based on the fusion of vehicle dynamic data (steering wheel, car velocity, and driver response time) with physiological responses (EDA, HRV, and respiration) to detect three stress levels of drivers. This study was conducted in a driving simulator and contained three driving sessions simulating different conditions, namely, relaxed, stress level 1, and stress level 2. Statistically significant differences in both physiological responses and vehicle dynamic data across the experiments were observed in the study. Previous findings in the literature (Rigas et al. 2012) also confirm that vehicle dynamic data can complement data from drivers' physiological responses in building a more accurate stress model. Vehicle dynamic data (steering wheel and speed) were used to detect the stressed or neutral state of drivers while talking on a cell phone (Bořil et al. 2012). Standard deviation and energies of high-frequency components of wavelet decomposition from the steering wheel and speed data were extracted in the study. The proposed model shows that the fusion of steering wheel and speed data with vocalization data in detecting the stress level of drivers is promising (achieved $88.2 \%$ accuracy).

3.3.4 Summary. The physical response data used to detect driver stress, presented in Sections 3.3.1 to 3.3.3 and in Table 2, show that although physical responses are measured using contactless methods, which are recommended over intrusive methods, they are used far less than physiological signals in this domain and need to be investigated more.

\subsection{Contextual Data}

Any stressful driving situation contains contextual parameters and stressors that induce a certain level of stress in the driver. Contextual parameters that can affect drivers' stress and performance are known as vehicle crash factors (Hennessy et al. 2000; Hill and Boyle 2007; Tchankue et al. 2013). Measuring contextual parameters along with the other stress responses can be beneficial for building a more accurate driver stress level detection system. Generally, contextual parameters can be divided into two categories: internal and external.

3.4.1 Internal Parameters. A driver's internal or individual parameters that can easily affect stress levels are important contextual data. A driver's mood can influence his or her brain's analysis of driving stressors, so stress is affected by whether that mood is positive or negative. Some studies demonstrated that negative moods, like aggression or frustration, can increase the risk of vehicle crashes (Hemenway and Solnick 1993; Underwood et al. 1999; Deffenbacher et al. 2003). Other studies showed that driver mood can be affected by different factors, such as driving at a certain time of a day, drinking coffee before driving (Singh et al. 2013), having a stressful day at the office (Matthews et al. 1998; Hennessy et al. 2000), and continually experiencing stressful events while driving (Hennessy et al. 2000). Another individual parameter, which has a high impact on perceived level of stress, is driver personality. Eysenck's personality questionnaire (EPQ) defines neuroticism/stability, psychoticism/sociability, and extraversion/intraversion as three major personality dimensions (Eysenck and Eysenck 1975). Neuroticism can increase driver stress level directly (Matthews et al. 1991) in that the perceived stress level by drivers who score high on neuroticism, in any stressful driving situation, may be higher than average. Psychoticism is closely linked to lacking in empathy (Eysenck and Eysenck 1975), so drivers who score high on psychoticism have a low susceptibility to stress (Matthews et al. 1991; Furnham and Saipe 1993). The level 
of perceived stress by this group of drivers, in any driving stressful situation, may be lower than average, which may explain their apparent tendency to exhibit risky driving behaviors (Sümer 2003). The relationship between driver stress and extraversion/intraversion is strongly dependent on the kind of driving situation that induces stress (Matthews et al. 1991); thus, this personality dimension cannot be considered as a general parameter that affects drivers' stress levels. Other individual factors such as age, gender, and crash history can also affect driver stress (Hill and Boyle 2007).

3.4.2 Environmental Parameters. Driver stress is also affected by environmental parameters such as driving in heavy traffic, heavy rain, icy roads, nighttime driving, and being trapped behind a slow-moving vehicle (Hill et al. 2007; Rigas et al. 2011), as well as family- and work-related issues (Gulian et al. 1989; Novaco et al. 1990). These environmental parameters can be divided into four groups of data: (1) weather-related conditions, (2) visibility-related conditions, (3) driverenvironment interactions (Hill and Boyle 2007), and (4) driving routes (Hennessy et al. 2000). (See Table 3.) Hill et al. (2007) used environmental parameters related to visibility-related conditions, weather-related conditions, and driver-environment interaction, along with internal parameters, to evaluate drivers' stress. The results show that the extracted parameters from the weather-related conditions and driver-environment interaction groups are more correlated to high levels of drivers' stress compared to the visibility-related conditions.

3.4.3 Summary. The studies of contextual data related to driver stress, presented in Sections 3.4.1 and 3.4.2 and in Table 3, show that collecting contextual data can be done in a low-cost and unobtrusive way with no extra expensive equipment, and that such data can provide useful information about driver stress levels. Incorporating this data into a driver stress detection system is recommended since it can help improve the performance of the system.

\section{FRAMEWORK APPROACH}

The focus of this article is to review state-of-the-art techniques and systems to detect driver stress levels. To structure this review, a general framework for an automatic and continuous driver stresslevel detection model is presented in Figure 1. The best modalities for data collection (signal acquisition) are reviewed in Section 3. Data analysis is a key approach for evaluating stress after collecting data from different modalities. Data analysis contains preprocessing, feature generation, feature selection, feature reduction, and machine-learning steps. This process helps to identify the relationship between stress and data modalities. Stress evaluation needs to take advantage of each of these steps to make the stress recognition process partially or fully automated, and also to explore the hidden related patterns within a large amount of data and implicit correlations. It is therefore of great interest to use different methods and investigate their roles in this domain to get a desirable outcome. The reviewed studies in this section are reported in more detail in Table 6.

\subsection{Preprocessing}

The first critical step in modeling driver stress levels is preprocessing and noise reduction of input data. Data can easily become corrupted by instrumentation and magnetic noise, body movement, and poor electrode skin contact. Preprocessing techniques remove all unwanted artifacts and provide purer data for the feature extraction step. This issue has been addressed by several studies in the domain of driver stress-level detection.

Baseline wander, muscle noise, and electrode misplacement are the most frequent sources of noise in ECG data, leading to detection of false-positive stressful events. A low-pass Butterworth filter can be used to remove baseline wander and muscle noise in drivers' ECG signals (Rigas et al. 2011). Extremely noisy blocks of bus drivers' ECG signals were detected and removed using a 
Table 1. Physiological Signals and Related Features Used to Analyze Driver Stress

\begin{tabular}{|c|c|c|}
\hline Signal & Reference & Feature \\
\hline $\mathrm{ECG}$ & $\begin{array}{l}\text { Healey and Picard (2000), Healey and Picard } \\
\text { (2005), Yamakoshi et al. (2007), Katsis et al. (2008), } \\
\text { Rigas et al. (2008), Yamakoshi et al. (2008), Akbas } \\
\text { (2011), Rigas et al. (2011), Deng et al. (2013), } \\
\text { Wang et al. (2013), Singh et al. (2013), Munla et al. } \\
\text { (2015), Rodrigues et al. (2015), Lanatà et al. (2015), } \\
\text { and Heikoop et al. (2017) }\end{array}$ & $\begin{array}{l}\text { Heart rate (HR): mean, standard deviation (SD) } \\
\text { PPG: mean, variance, SD, signal energy, time duration, } \\
\text { bandwidth, time-bandwidth product dimensionality, cardiac } \\
\text { output, PPG instantaneous, pulse height, PPG rise, PPG fall, } \\
\text { PPG cardiac, NPV } \\
\text { Heart rate variability (HRV) (time domain): mean of the } \\
\text { first difference (MFD), mean of R-to-R intervals (MRR), } \\
\text { SDRR, MNN, SDNN, RMSSD, pNN20, pNN50, NN50, } \\
\text { triangular interpolation of the NN interval histogram } \\
\text { (TINN), heart rate variation from baseline } \\
\text { - HRV (frequency domain): LF/HF ratio, HF, LF, very low } \\
\text { frequency (VLF), total power (TP) } \\
\text { - HRV (nonlinear analysis): sample entropy, detrended } \\
\text { fluctuation analysis (DFA), DFA- } \alpha 1 \text { and DFA- } \alpha 2, \text { SD1, SD2 } \\
\text { HRV (time-frequency domain analysis): VLF, LF, and } \\
\text { HF by using wavelet and short time Fourier transform } \\
\text { (STFT) analysis }\end{array}$ \\
\hline EDA & $\begin{array}{l}\text { Avcı Akbaş and Yüksel (n.d.), Healey and Picard } \\
\text { (2000), Healey and Picard (2005), Rigas et al. } \\
\text { (2008), Katsis et al. (2008), Akbas (2011), Rigas } \\
\text { et al. (2011, 2012), Deng et al. (2013), Singh and } \\
\text { Queyam (2013b), Singh et al. (2013), Lanatà et al. } \\
\text { (2015), and Ollander et al. (2017) }\end{array}$ & $\begin{array}{l}\text { Mean, variance, SD, mean amplitude, peak } \\
\text { rise time, peak amplitude, half-recovery, peak energy, rise } \\
\text { rate average, decay rate average, percentage decay, number of } \\
\text { peaks, rate of SCR, first absolute difference (FAD), MFD, mean } \\
\text { rise duration, number of SCR, latitude, the amplitude sum of } \\
\text { SCRs (AmpSum), the phasic area under curve, the max } \\
\text { phasic amplitude, mean of SCR, variance of SCR, mean of } \\
\text { SCL, variance of SCL, number of the nonspecific response, } \\
\text { frequency, magnitude, duration, area of orienting } \\
\text { responses, sum of frequency of occurrence (SF), sum of } \\
\text { durations, sum of magnitudes (SM), sum of the estimated } \\
\text { areas (SA), mean of positive derivative, mean of absolute } \\
\text { derivative, proportion of positive samples in derivative, } \\
\text { number of local maxima }\end{array}$ \\
\hline $\begin{array}{l}\text { Respiratory } \\
\text { Response }\end{array}$ & $\begin{array}{l}\text { Healey and Picard (2000), Katsis et al. (2008), } \\
\text { Katsis et al. (2011), Rigas et al. (2011), Akbas } \\
\text { (2011), Deng et al. (2012), Rigas et al. (2012), } \\
\text { Lanatà et al. (2015), and Ollander et al. (2017) }\end{array}$ & $\begin{array}{l}\text { Time Domain: mean of RESP, SD of RESP, average number } \\
\text { of contractions/minute, RSPR, HR/RESP ratio, dominant } \\
\text { respiration frequency, PPG respiratory rate (PPGRSP); max, } \\
\text { min, and mean breathing amplitude; standard error of the } \\
\text { mean (SEM), difference between max and min value of } \\
\text { breathing amplitude, skewness, kurtosis, entropy of the } \\
\text { resulting spectrum, MFD, mean of the second difference } \\
\text { (MSD); standard deviation of the first difference (SDFD), } \\
\text { standard deviation of the second difference (SDSD); second } \\
\text { difference breathing amplitude (SDBA), maximal } \\
\text { respiration-mean of respiration (range) } \\
\text { Frequency Domain: RESP frequency (0-0.1), RESP } \\
\text { frequency (0.1-0.2), RESP frequency }(0.2-0.3), \text { RESP } \\
\text { frequency (0.3-0.4) }\end{array}$ \\
\hline EMG & $\begin{array}{l}\text { Katsis et al. (2008), Soman et al. (2014), and Zheng } \\
\text { et al. (2015) }\end{array}$ & Mean, RMS, ZCR, RMQ \\
\hline ST & $\begin{array}{l}\text { Yamakoshi et al. (2007), Shastri et al. (2008), and } \\
\text { Yamakoshi et al. (2008) }\end{array}$ & $\begin{array}{l}\text { Finger temperature (Tsf), nose temperature (Tsn), cheek } \\
\text { temperature (Tsch), jaw temperature (Tsj), forehead } \\
\text { temperature (Tsfh) }\end{array}$ \\
\hline Blood Pressure & Yamakoshi et al. (2008) & $\begin{array}{l}\text { Systolic blood pressure, mean blood pressure, diastolic blood } \\
\text { pressure }\end{array}$ \\
\hline Pupil Dilation & Pedrotti et al. (2014) & $\begin{array}{l}\text { First different pupil diameter, wavelet decomposition detail } \\
\text { for pupil diameter }\end{array}$ \\
\hline
\end{tabular}

standard deviation filter method (Rodrigues et al. 2015). For HRV signals, R peak detection algorithms may make mistakes in calculating interbeat (RR) intervals from ECG signals. Lanatà et al. (2015) applied an adopted piecewise cubic spline interpolation method to correct these technical artifacts in RR intervals and then manually checked and removed physiological artifacts, which include ectopic beats and arrhythmic events, in RR intervals.

In order to compensate for any artifacts in drivers' EDA signals generated by baseline wander, sensor, and body movement, different filter methods have been used in the literature. A smoothing filter (moving average) was used to find high-frequency noises in drivers' EDA signals (Rigas 
Table 2. Physical Signals and Related Features Used to Analyze Driver Stress

\begin{tabular}{|c|c|c|}
\hline Signal & Reference & $\begin{array}{c}\text { Feature } \\
\end{array}$ \\
\hline Face & Gao et al. (2014) & $\begin{array}{l}\text { Facial Expression (FER): local DCT feature, } \\
\text { local descriptors. }\end{array}$ \\
\hline $\begin{array}{l}\text { Vehicle } \\
\text { Dynamic Data }\end{array}$ & $\begin{array}{l}\text { Bořil et al. (2012), Rigas } \\
\text { et al. (2012), Lanatà } \\
\text { et al. (2015), and Lee } \\
\text { et al. (2017) }\end{array}$ & $\begin{array}{l}\text { Steering-wheel angle (SWA): wavelet } \\
\text { decomposition energy for SWA; standard } \\
\text { deviation of SWA (SDSWA), SDSWA rate, } \\
\text { sample entropy; area under the SWA curve; } \\
\text { number of sign changes of SW; mean and SD of } \\
\text { SWA differences between two consecutive } \\
\text { skids; reaction time; SWA first peak; SWA } \\
\text { second peak; first response time of SWA, first } \\
\text { ability time of SWA } \\
\text { - Steering wheel motion (SWM) captured by } \\
\text { orientation sensor (Y-axis): mean, SD, } \\
\text { variance, average squared power, median, root } \\
\text { mean square, range difference max and mean, } \\
\text { sum power spectrum energy (0-8Hz), mean } \\
\text { power frequency (0-8Hz), Shannon entropy } \\
\text { energy (0-8Hz), peak power frequency } \\
\text { (0-8Hz), median power frequency (0-8Hz), } \\
\text { percentage of point outside the control eclipse, } \\
\text { weighting function outside the control eclipse } \\
\text { Speed: wavelet decomposition energy, sample } \\
\text { entropy, SD, average } \\
\text { Deceleration: average } \\
\text { - Heading Change: average magnitude in } \\
\text { heading changes } \\
\text { Throttle: average } \\
\text { Engine performance: Engine's rounds per } \\
\text { minute (RPM) } \\
\text { Overtaking: average }\end{array}$ \\
\hline vocalization & $\begin{array}{l}\text { Fernandez and Picard } \\
\text { (2003), Boril et al. } \\
\text { (2010), and Bořil et al. } \\
\text { (2012) }\end{array}$ & $\begin{array}{l}\text { Mel frequency cepstral coefficients (MFCC), } \\
\text { perceptual linear prediction cepstral } \\
\text { coefficients (PLPCC), mean and SD center } \\
\text { bandwidths and frequencies of the first four } \\
\text { formants (F1, F2, F3, F4) extracted from voiced } \\
\text { speech segments }\end{array}$ \\
\hline
\end{tabular}

et al. 2008). These noises were caused by the movements of the EDA sensors. In another study, a Butterworth filter (low-pass filter $1 \mathrm{~Hz}$ and high-pass filter $0.1 \mathrm{~Hz}$ ) was used to remove baseline wander in drivers' EDA signals (Rigas et al. 2011).

Generally, drivers' respiration signals are observed to be less noisy compared with other physiological signals. Sudden body movement is the main source of noise in this signal. A moving-average filter and finite impulse response filter (10th-order low-pass) were applied sequentially to drivers' respiration signals to detrend the baseline and remove noises caused by body movements from each of the signals (Lanatà et al. 2015). 
Table 3. Contextual Data and Related Features Used to Analyze Driver Stress

\begin{tabular}{|c|c|c|c|}
\hline Context Type & Reference & Context Name & $\begin{array}{c}\text { Data Collection } \\
\text { Method }\end{array}$ \\
\hline Internal/individual & $\begin{array}{l}\text { Matthews et al. } \\
(1991), \text { Hennessy } \\
\text { et al. (2000), Hill and } \\
\text { Boyle (2007), and } \\
\text { Cantin et al. (2009) }\end{array}$ & $\begin{array}{l}\text { Personality traits, mood, gender, age, gender, number of } \\
\text { crashes, commuting, alcohol consumption per week, } \\
\text { driving behavior history, years driving experience }\end{array}$ & Questionnaire \\
\hline External/environmental & $\begin{array}{l}\text { Novaco et al. (1990), } \\
\text { Hill and Boyle } \\
\text { (2007), Rigas et al. } \\
\text { (2011), and Miller } \\
\text { and Boyle (2013) }\end{array}$ & $\begin{array}{l}\text { Route data: direction of travel, driving on mountain } \\
\text { roads } \\
\text { Weather-related conditions: heavy rain, no rain, rain, } \\
\text { snow, cold weather, icy and slippery roads } \\
\text { Visibility-related conditions: nighttime driving, road } \\
\text { visibility, driving behind large trucks } \\
\text { Driver-environment interactions: braking due to } \\
\text { slower drivers, drivers' work-related problems, driver's } \\
\text { family disturbances, traffic density }\end{array}$ & $\begin{array}{l}\text { Questionnaire, } \\
\text { mobile GPS, vehicle } \\
\text { GPS, forward- } \\
\text { looking camera, } \\
\text { CAN-bus system }\end{array}$ \\
\hline
\end{tabular}

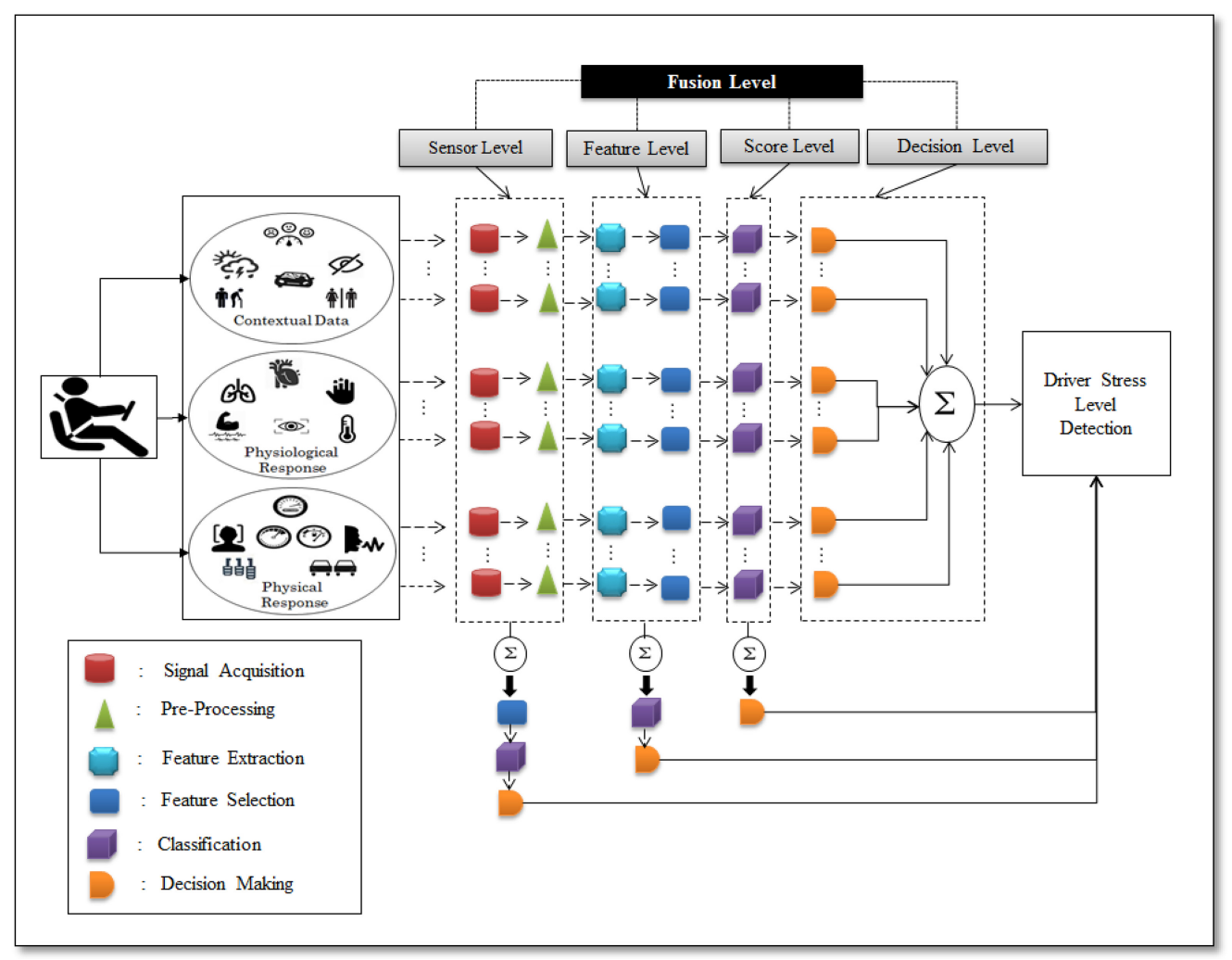

Fig. 1. Driver stress-level detection framework.

Motion artifact in drivers' PPG signals were filtered by applying a one-dimensional median filter with an order of four samples (Singh et al. 2013). Next, the lost signal peaks were reconstructed using a cross-correlation detection method. The authors detrended the reconstructed PPG signal to remove any baseline drifts from the signal as well. In this study, PPG signal spikes and impulse noises were filtered by applying another one-dimensional median filter of size 3 . 
In another study, body movement noises in drivers' facial EMG signals were removed by using a Butterworth low-pass filter $(500 \mathrm{~Hz})$ (Katsis et al. 2008). Pose variation as a source of noise in drivers' facial expression data was removed using an adapted least square method (Gao et al. 2014). A fast Fourier transformation technique, adapted to a based noise cleaning approach, was used to remove substantial noise in the supraorbital region from drivers' temperature signals (Shastri et al. 2008). Borril et al. (2012) used a full-wave spectral subtraction method to reduce noise in drivers' vocalization signals. In addition, other studies used KNN algorithms to correct missing data (Rigas et al. 2011) and normalization techniques to remove the role of intersubject variability (Singh et al. 2013; Rodrigues et al. 2015; Lanatà et al. 2015).

\subsection{Feature Extraction}

Feature extraction aims to decrease noise and redundancy from input data and operate on the significant information of the data. Several techniques have been employed to extract relevant features from the time, frequency, and time-frequency domains of primary nonstationary data to indicate driver stress levels. The features from different modalities (physiological, physical, and contextual data) extracted to analyze driver stress are reviewed in Tables 1, 2, and 3.

The extracted features from the physiological signals, reviewed in the previous section, are summarized in Table 1. Most of the physiological features were extracted from time and frequency domains. Further analyses (nonlinear and time-frequency) were also applied to extract more complicated features from the ECG signal to detect drivers' stress.

Table 2 sets out the features extracted from the physical signals reviewed in Section 3.3. In this table, most of the vocalization features were extracted from time-frequency and frequency domains, while the extracted features from vehicle dynamic data are from time, nonlinear, and time-frequency analyses. The features extracted from contextual data (both individual and environmental conditions) are presented in Table 3.

To investigate the general quality features for driver stress recognition based on real-time and simulated driving scenarios, and which features are repeated more frequently or performed more successfully in driver stress classification, we summarized the most widely used data and their commonly extracted features in this domain. (See Table 4.) Based on the literature, among the physiological signals, ECG, EDA, respiration, and EMG signals show the most remarkable data, and their correlated features are presented in the table. It has been confirmed that vehicle dynamic data can complement physiological responses in building a more reliable and accurate stress model. The common extracted features from this data are from vehicle steering-wheel angle, speed, deceleration, heading change, throttle, and engine performance data. Among the contextual parameters, the features extracted from individual parameters, weather-related conditions, and driverenvironment interactions are shown to be more correlated to driver stress levels (Hill et al. 2007; Rigas et al. 2011).

\subsection{Feature Selection}

Increasing the number of extracted features causes high computational costs and also introduces unnecessary noise, which reduces the accuracy of classification algorithms (Kira and Rendell 1992). A feature space with high dimensions impedes target classification learning. Feature selection methods help to reduce redundant features and negative effects of high dimensionality.

Different feature selection techniques search for the best subset of features based on evaluation functions to enhance overall classifier performance. Generally these methods are divided into four groups, namely, filter, wrapper, embedded (Kudo and Sklansky 2000; Alba et al. 2007; Nakisa et al. 2017), and hybrid methods (Min and Fangfang 2010; Wang and Liu 2016). In the domain of driver stress detection, these methods are employed in some studies to improve performance. 
Table 4. The Most Widely Used Data and Their Commonly Extracted Features to Build Driver Stress Recognition Model

\begin{tabular}{|c|c|c|}
\hline Data & $\begin{array}{l}\text { Experimental } \\
\text { Setting }\end{array}$ & Features \\
\hline \multirow[b]{2}{*}{ ECG } & Simulator & $\begin{array}{l}\text { Mean, SD, SDNN, RMSSD, pNN50, TINN, MRR, } \\
\text { SD of RR, LF/HF ratio, LF, VLF }\end{array}$ \\
\hline & Real Time & $\begin{array}{l}\text { Mean, SD, MNN, SDNN, RMSSD, pNN20, pNN50, } \\
\text { NN50, MFD, MRR, LF/HF ratio, LF, VLF, HF, TP }\end{array}$ \\
\hline \multirow[b]{2}{*}{ EDA } & Simulator & $\begin{array}{l}\text { Mean, FAD, mean of SCR, variance of SCR, } \\
\text { AmpSum }\end{array}$ \\
\hline & Real Time & $\begin{array}{l}\text { Mean, mean amplitude, peak rise time, peak } \\
\text { amplitude, half-recovery, peak energy, rise rate } \\
\text { average, decay rate average, percentage decay, } \\
\text { number of peaks, rate of SCR, FAD, MFD, } \\
\text { frequency, magnitude, duration, area of orienting } \\
\text { responses, SF, sum of duration, SM, mean of } \\
\text { positive derivative, mean of absolute derivative, } \\
\text { proportion of positive samples in derivative, } \\
\text { number of local maxima }\end{array}$ \\
\hline \multirow[b]{2}{*}{ Respiration } & Simulator & $\begin{array}{l}\text { Mean of RESP, SEM, MFD, MSD, SDFD, SDBA, } \\
\text { difference between max and min value of } \\
\text { breathing amplitude, kurtosis, RESP frequency } \\
(0-0.1), \text { RESP frequency }(0.2-0.3) \text {, RESP frequency } \\
(0.3-0.4)\end{array}$ \\
\hline & Real Time & $\begin{array}{l}\text { Mean of RESP, SD of RESP, RSPR, HR/RESP ratio, } \\
\text { PPGRSP; max, min, and mean breathing } \\
\text { amplitude, MFD, maximal respiration-mean of } \\
\text { respiration (range), RESP frequency }(0-0.1) \text {, RESP } \\
\text { frequency }(0.1-0.2)\end{array}$ \\
\hline \multirow[t]{2}{*}{$\begin{array}{l}\text { Vehicle } \\
\text { Dynamic Data }\end{array}$} & Simulator & $\begin{array}{l}\text { - Steering-wheel angle (SWA): area under the SWA } \\
\text { curve; number of sign changes of SW; Mean and } \\
\text { SD of SWA differences between two consecutive } \\
\text { skids; reaction time; SWA first peak; SWA second } \\
\text { peak; first response time of SWA, first ability time } \\
\text { of SWA }\end{array}$ \\
\hline & Real Time & $\begin{array}{l}\text { Average of vehicle speed, average of vehicle } \\
\text { deceleration, average magnitude in vehicle } \\
\text { heading changes, average of throttle, RPM }\end{array}$ \\
\hline \multirow[b]{2}{*}{$\begin{array}{l}\text { Contextual } \\
\text { data }\end{array}$} & Simulator & $\begin{array}{l}\text { - Age, years driving experience, commuting, alcohol } \\
\text { consumption per week }\end{array}$ \\
\hline & Real Time & $\begin{array}{l}\text { - Age, gender, number of crashes, weather condition } \\
\text { (no rain, rain, heavy rain), snow, cold weather, icy } \\
\text { and slippery roads, braking due to slower drivers, } \\
\text { traffic density }\end{array}$ \\
\hline
\end{tabular}


4.3.1 Filter Methods. Filter methods use ranking techniques-which are used for their simplicity and good success in different applications-to order the features. Ranking techniques score each feature based on its relevance to an outcome variable and use a threshold to remove the features below the threshold. Several studies have applied filtering methods to explore the features most relevant to different levels of drivers' stress. Deng et al. (2013) extracted 22 features from drivers' EDA, EMG, respiration, and HR signals and then used a filtering method, which first measured the efficiency of the features separately and then calculated diversity among the features. The feature sets selected by this method were then combined using a combinatorial fusion method to detect three stress levels of drivers. The results show that the proposed methods increase the detection rate. In another study, difference in the area under curve (DAUC) was used as a feature selection method to rank 22 features extracted from drivers' EDA, HRV, respiration, head movement, weather, traffic, and visibility condition data (Rigas et al. 2011) and evaluate the discrimination ability of the features between two stress classes (normal/stressed). For each of the class labels, the DAUC values of the features were measured and then averaged, and these average values were sorted to obtain a ranking list for the features. Furthermore, some studies (Singh and Queyam 2013a, 2013b) used the Pearson product-moment correlation coefficient method to remove redundant extracted features. Lanatà et al. (2015) extracted 43 features from EDA, HRV, respiration, steering-wheel angle, car velocity, and drivers' response time signals, and then used a Friedman test followed by a post hoc test (Bonferroni adjustment) to find the significant statistical features $(\mathrm{p}<0.05)$. The test selected 16 features from all the extracted features and put them in a group (Group $\beta$ ). Another feature group (Group $\alpha$ ) contained all of the 43 features extracted from HRV, EDA, respiration (RSP) signals, and the vehicle dynamic data, while a third feature group (Group $\gamma$ ) contained all features extracted from the EDA signals. The features of Groups $\alpha, \beta$, and $\gamma$ were used separately to detect drivers' stress levels. The best stress model was built by using the selected features of group $\beta$, which had an accuracy greater than 90\%. Ollander et al. (2017) used four different filter methods-Pearson's linear correlation coefficient, Spearman's rank correlation, Fisher score, and receiver operating characteristic-to score 14 extracted features from ECG, EDA, respiration, and EMG signals. Then the mean value of all ranks for each feature was calculated to distinguish two groups of driver states: rest versus stress, and low stress versus high stress. Based on the mean value, EDA and ECG features are shown to be more correlated to classifying rest from stress states, while EDA and respiration features can distinguish low and high stress more accurately.

4.3.2 Wrapper Methods. Wrapper methods find heuristically suboptimal feature subsets by applying search algorithms. SFFS is a wrapper method used in the literature that iteratively finds the near-optimal subset of the features. This method first starts with a null subset and, based on defined criteria, evaluates the features, and then adds the features that satisfy the criteria to the subset. The algorithm is designed to modify the criteria if some features are excluded. In this case, based on the criteria, the features in the subset will be ordered and the worst feature is removed from the subset. By using this dynamic process, increasing and decreasing the features in the subset, the SFFS method gradually reaches the near-optimal feature set. Healey and Picard (2000) applied SFFS on 11 features that were extracted from drivers' ECG, EDA, and respiration signals. Seven features were selected by SFFS and then fed to a KNN classifier. The recognition rate of the proposed model was reported as $88.6 \%$. Ollander et al. (2017) used an exhaustive feature selection method to find the best subset of features from each set of ECG, EDA, respiration, and EMG signals. Next, to find the optimal signal combination, an exhaustive search method was applied on the selected features from the signals. The result indicated that the combination of ECG, EDA, and respiration signals to distinguish drivers' rest from stress has the optimal performance (accuracy: 87.3\% \pm 2.8 ) among 
different signal combinations. In another study, SFFS was applied on 20 features extracted from drivers' PPG signals and steering-wheel motion data (Lee et al. 2017). The features were separately fed to an SVM-Gaussian kernel function classifier to evaluate their performance. Using the SFFS method, the top eight of 20 features were selected as the optimal subset, with accuracy over $95 \%$, to assess drivers' stress. The result indicates that among the eight selected features, frequencydomain features are more correlated to drivers' stress than time-domain features.

4.3.3 Embedded Methods. Embedded methods seek to decrease the computation time for training different subsets computed in the wrapper methods (Chandrashekar and Sahin 2014). Wang et al. (2013) applied a feature-based transformation recognition technique, which consists of feature extraction, selection, and reduction steps. They applied kernel-based class separability (KBCS) as a feature selection method, and PCA and LDA as feature reduction methods, to select the most relevant and important drivers' ECG features, and then a KNN classifier was used to build a stress model. The proposed model shows an acceptable performance compared to the same studies that used multiple signals and features.

4.3.4 Hybrid Methods. Hybrid methods try to combine filter and wrapper methods to increase the efficiency and accuracy of feature selection. The hybrid method is used in a driver stress level detection model to enhance the performance of the model (Singh et al. 2013). The filter method was a combination of entropy and variance techniques, whereas the wrapper method was an integration of two sequential feature selection techniques, namely, sequential backward selection (SBS) and sequential forward selection (SFS). First, the filter selection techniques were applied separately to find a relevant subset of the features. Next, the results were subjected to bitwise conjunction ("ANDed") to provide a new subset of the features. In the next phase, applying the wrapper method, the SBS and SFS techniques selected a number of features together. Finally, the results from both techniques were compared and 27 features were selected. In addition to this result, they applied an ad hoc feature mask to ensure that no significant clinical features were lost, and the results were subjected to bitwise disjunction ("ORed"), providing 30 features.

\subsection{Machine-Learning Approaches}

Machine-learning approaches are employed to build a model that can detect or predict the stress levels of drivers (supervised learning) or cluster them (unsupervised learning). These methods try to explore the patterns most related to drivers' stress levels. The classifiers used in this domain range from linear classifiers, such as linear mixed model and linear SVM; to nonlinear classifiers, such as ANN and SVM with polynomial and Gaussian kernels; to probabilistic classifiers, such as naïve Bayes classifier (NBC). The most common and important classification techniques used for driver stress level detection are tabulated in Table 5.

4.4.1 Artificial Neural Network. An ANN simulates brain function through mathematical functions (Fyfe 2005) and aims to solve machine-learning problems by combining a large amount of processing elements (neurons) that are highly interconnected and work together (Huang et al. 2006). Generally, an ANN consists of three types of artificial neurons-input, hidden, and output neurons-which are organized in layers. Each neuron is connected to all the neurons in its layer and a value (weight) is assigned to the connection between them. The neurons also send an activation value to each of the neurons in the next layer. This value is measured based on their output value and the weight between them and their connected neuron in the next layer. Therefore, the connection between the neurons in an ANN can be explained as follows: (1) input values are assigned to the input neurons; (2) in the hidden layers, each neuron sums the received activation values from its connected neurons and then determines its output value by using a transfer 
function; (3) a similar process is done in the output layer to generate the output values of the neural network. An ANN classifier was used to build a personal/general driver stress-level detection model through physiological signals. A four-way binary neural network classifier was used by Pedrotti et al. (2014) to classify four stress levels of drivers. Each of the binary neural networks contained two hidden layers and discriminated one of the stress levels from the rest. In the test phase, new samples were scored for each of the stress levels by using the trained classifiers. Finally, the stress levels of the test samples were determined based on their highest score. The precision of this method was reported as $79.2 \%$.

4.4.2 Layer Recurrent Neural Network. LRNNs have a similar mechanism to feed-forward neural networks, but they have feedback connections to capture dynamic temporal patterns (Medsker and Jain 1999). In recurrent neural networks, inputs are dependent on each other and each output is the function of its corresponding inputs and previous states (Liu and Wang 2008). LRNNs have internal feedback loops in each hidden neuron, which allows them to store the previous states. In this process, the output of each hidden neuron is stored in a context unit, then fed to the hidden neuron after a predetermined delay. Based on the latency in drivers' responses to stressful driving situations, an LRNN can handle these delays by using its context units. To this end, this dynamic neural network and six different static neural networks were used separately to detect three stress levels of 19 drivers from their physiological data (Singh et al. 2013). The performances of seven classifiers were compared based on a proposed parameter called a desirability measure. This parameter was calculated based on the precision, sensitivity, specificity, F-measure, and Gmean values to evaluate the performance and consistency degree of each classifier. The result showed that LRNN has the best performance among all machine-learning approaches.

4.4.3 Support Vector Machines. Another classification method is SVM (Hsu et al. 2003), which has been used for high-dimensional problems. This algorithm is a discriminative classifier that iteratively searches to find the optimal hyperplanes to separate training samples accurately. The selection of these hyperplanes is based on two criteria: first, the hyperplanes must separate training samples accurately, and second, there must be a greatest possible distance between support vector points and the hyperplanes. SVM deals with nonlinear problems by using kernel functions that map nonlinear separable data into high-dimensional space, then finds the optimal hyperplanes. SVM classifiers, with three different kernel functions-linear, polynomial, and Gaussianwere used to detect stress of 28 drivers using their PPG signals and steering-wheel motion data (Lee et al. 2017). The highest accuracy (95.38\%) was obtained using an SVM-Gaussian kernel function classifier. The SVM-Gaussian kernel function classifier was used to distinguish low and high stress levels of 14 drivers using their ECG and EMG signals (Soman et al. 2014). The sensitivity, accuracy, classification rate, and specificity of this model were reported as $100 \%$. Katsis et al. (2008) collected ECG, EMG, EDA, and RSP signals of 10 drivers and classified them into four stress levels using an SVM classifier (79.3\% accuracy).

4.4.4 Bayesian Networks. A Bayesian network (BN) is designed as a probabilistic model that allows representation and reasoning with uncertain data (Friedman et al. 1997). It is a graphical model that contains nodes and links. Each node represents a feature and has a set of possible values. The link between two nodes represents a direct dependency between their features, which are quantified by conditional probability. A naïve Bayes classifier (NBC) is a special variant of $\mathrm{BN}$ in which class nodes have no parent and there is no link between nodes related to features, and the feature nodes just link to the class nodes. An NBC offers a competitive performance against other sophisticated classification methods such as decision trees and ANN (John and Langley 1995; Theodoridis and Koutroumbas 2006). Furthermore, this method can handle high-dimensional data 
easily, due to its short training time (Hand et al. 2001). Rigas et al. (2012) collected ECG, EDA, and RSP signals along with some vehicle dynamic data from 13 drivers, and then built two different BN models to detect drivers' stress. The first model (BN1) used the physiological signals to detect drivers' stress and achieved $82 \%$ accuracy on the test dataset. The second model (BN2) merged BN1 with two NBCs to make a model that supported mutual relations between stress and event detection. The NBCs were used to detect overtaking and hard braking events by using the vehicle dynamic data. BN2 achieved $96 \%$ accuracy on the test data.

4.4.5 Dynamic Bayesian Network. A dynamic Bayesian network (DBN) is represented as a probabilistic framework for uncertainty knowledge information (Murphy 2002). This method is an extended version of $\mathrm{BN}$ for dynamic processes. Each node represents a feature value in a specific time, and the relationships between nodes are determined based on their occurrence times. Rigas et al. (2008) applied a three-layer DBN model to detect three stress levels of a driver: natural, low, and medium-high. This model was built using the driver's ECG and EDA signals, and achieved an overall accuracy of $71.2 \%$. In the study, the DBN architecture involved layers of risks, diseases, and symptoms, inspired by medical expert systems. Driving events were used in the risk layer, the driver's stress levels were used in the diseases layer, and extracted features from the driver's ECG and EDA signals were used in the symptoms layer.

4.4.6 Decision Tree. A decision tree (DT) is a popular classifier that represents decision rules (Safavian and Landgrebe 1990). Features are represented as nodes and class labels are represented as leaf nodes. The structure of a DT is determined based on dataset homogeneity. This means that features are inserted into the tree nodes based on their ability to split training samples into their actual classes. To this end, the best splitter or feature is selected by using a greedy search algorithm and inserted in the root node. Then the training samples, based on the feature values, are divided into various branches. If all the subsamples in a branch belong to a specific class label, it leads to a leaf node and the branch is marked to be not extended anymore. Otherwise, DT finds the best splitters or feature for subsamples in the branch to divide the samples into more branches. This process will be repeated until there is no branch to be extended. In one study, DT and tree-augmented NBC were hybridized to classify four stress levels of drivers (Katsis et al. 2011). The accuracy of this method was reported as $71.9 \%$, measured using a 10 -fold cross-validation technique.

4.4.7 Adaptive Neuro-Fuzzy Inference System. An adaptive neuro-fuzzy inference system (ANFIS) classifier (Jang 1991) is counted as a powerful discriminator by integrating an ANN (multilayer feed-forward network) and fuzzy reasoning. This algorithm combines the advantages of these two methods to solve classification problems (Chang and Chang 2006). An ANFIS classifier is able to work with numerical data or experts' knowledge and extract fuzzy rules from them (Kia et al. 2006). An ANFIS classifier includes a premise and consequent parameters that together play a significant role in improving classifier performance. Katsis et al. (2008) used an ANFIS classifier to classify four emotion classes of drivers using physiological signals and achieved $76.7 \%$ accuracy. The classifier was based on the combination of a least squares (forward pass) and gradient descent method (backward pass). This combination was selected due to its high efficiency in tuning the premise and consequent parameters to improve the performance of the ANFIS classifier. In the forward-pass step, the ANFIS consequent parameters were optimized by applying the least squares method, and then in the backward-pass step, its premise parameters were adjusted using the gradient descent method based on the fuzzy set.

4.4.8 Hidden Markov Model. The hidden Markov model (HMM) is a classifier that can work with a wide range of time-series data. In a simple Markov model, labels are the hidden states and observations linked to the labels are the visible states. This classifier predicts future states based 
on the following assumptions. The current observation is generated from a hidden state at time $t$. This hidden state, based on the Markov property, is dependent on the previous hidden state at time $t$-1. Therefore, an HMM classifier finds all possible sequences of labels for a sequence of observations and computes probability distributions over each of the sequences of labels, and then the best label sequence is selected to assign to that sequence of observations. Although HMM classifiers have been employed in different domains, they are well known in the speech recognition domain (Ghahramani 2001). Fernandez et al. (2003) used five different HMM classifiers to recognize four stress levels of drivers using their voice waveforms. The selected classifiers were HMM, autoregressive hidden Markov model (ARHMM), factorial hidden Markov model (FHMM), hidden Markov decision tree (HMDT), and a mixture of simple hidden Markov models (M-HMM). The best classification accuracy was obtained using the M-HMM classifier (51.22\%).

4.4.9 K-Nearest Neighbors. A KNN classifier is a simple and effective algorithm that uses a probabilistic approach to classify new samples based on a similarity measure (e.g., distance). In the training step, the algorithm only stores all samples in the training dataset. In the test step, for each test sample, the algorithm finds k most similar training samples (neighbors) and then selects the most frequent class label among them (Altman 1992). This label is assigned to the test instance as its class label. Although a KNN classifier is conventionally used in biometric and stress management systems, it is more computationally expensive than other classifiers. A KNN classifier was used to distinguish low, medium, high, and very high stress levels of 10 drivers using their ECG, EDA, EMG, and RSP signals (Healey and Picard 2000). The accuracy of this model was reported as $88.6 \%$. Wang et al. (2013) used a KNN classifier to discriminate two drivers' stress levels using ECG signals and achieved an averaged recognition rate of $97.78 \%$ (fivefold cross-validation).

4.4.10 Nearest Mean Classifier. A nearest mean classifier (NMC) is a simple and linear classification algorithm that is based on a template matching approach. This classifier discriminates new samples based on a similarity measure (e.g., distance). An NMC stores all samples in the training dataset. The mean point of each class label is determined based on the corresponding training samples. Then, the NMC assigns each test sample to a class label whose mean is closest to the test sample. Lanatà et al. (2015) used NMC, KNN, and multilayer perceptrons to detect three stress levels using physiological and physical data of 14 drivers. These classifiers are representative of template matching, probabilistic, and decision boundary construction approaches, respectively. To evaluate the classifiers' performance, a leave-one-subject-out cross-validation technique was employed. It has been shown that the NMC had the best performance, achieving over $90 \%$ accuracy.

4.4.11 Linear Discernment Analysis. A linear discernment analysis (LDA) is a linear classifier that aims to maximize class separability and reduce computational costs by projecting a $d$ dimensional sample onto a $k$-dimensional subspace (where $k<d$ ). LDA performs this approach in five steps: (1) computes a $d$-dimensional mean vector for each class label, (2) computes two $d * d$ dimensional scatter matrices called a between-class scatter matrix and a within-class scatter matrix, (3) computes the $d$-dimensional eigenvectors and corresponding $d$-dimensional eigenvalues for the calculated scatter matrices, (4) drops the $(d-k)$ eigenvectors with the lowest eigenvalues to reduce the dimensionality of the sample; (5) transforms the sample onto the reduced feature space. An LDA classifier was used to build a general/personal driver stress-level detection model through physiological signals and to classify drivers' stress into three levels (low, medium, and high) using their EDA, ECG, respiration, and EMG signals (Healey et al. 2005). The recognition rate of the proposed model was reported as $97.4 \%$. Urbano et al. (2017) collected ECG and EDA signals of six drivers and built three personal stress detection models for each driver using SVMlinear kernel function, SVM-quadratic kernel function, and LDA classifiers. The result indicated 
that the built models using the LDA classifier were almost better than the other models built by the other classifiers.

\subsection{Fusion Techniques}

Detecting driver stress level using different modalities may not attain perfect accuracy due to different issues such as nonideal machine-learning techniques and incomplete training, resulting in a false negative and false positive (Blasco et al. 2016). Combining different modalities can help to overcome some of these limitations and build a more accurate and reliable driver stress recognition model. Modality fusion is defined as the integration and combination of different types of data collected from a subject in order to detect different affects (Gunes and Pantic 2010). Modality fusion can be performed at any level of data analysis, such as sensor-level, feature-level, score-level, or decision-level fusion. Most of the reviewed studies used feature-level fusion techniques to build their driver stress-level detection model. In feature-level fusion, all the extracted features from each modality are combined into one vector for use in the next data analysis step. Combining different modalities at the feature level has been demonstrated to be an effective way to build a driver stress-level detection model (Healey and Picard 2005; Katsis et al. 2008; Rigas et al. 2012; Lanatà et al. 2015).

\subsection{Challenges for Framework Implementation and Evaluation}

In order to develop an automated system that proactively detects driver stress levels, data needs to be collected, transferred, preprocessed, reduced, integrated, and used to make the final decision automatically. To increase driving safety, this system needs to present a practical solution that continuously, unobtrusively, and automatically detects driver stress levels at an early stage. Reviewing the literature shows several methodological issues that hinder the implementation of such a system. These issues can be grouped into six major categories: (1) data quality, (2) dataset, (3) modality fusion, (4) data analysis, (5) system acceptability, and (6) driving scenario. Sections 4.6.1 to 4.6.6 discuss how these categories may be considered open research problems in the domain of driver stress-level detection.

4.6.1 Data Quality. Collecting high-quality data is essential to the success of any stress detection system. Therefore, data collection should be carried out meticulously to obtain data that is accurate, efficient, secure, relevant, timely, and appropriately represented. Generally, the quality of data can be corrupted by different issues such as inadequate sampling rates of recording devices and the presence of noise in the data. Some studies employed recording systems with high sample rates to overcome this issue (Healey and Picard 2000; Lanatà et al. 2015). However, it should be noted that any real-world driving scenario will potentially cause noise and artifacts in data. Although different filtering methods have been applied to cope with this issue (see Section 4.1), more accurate and reliable algorithms are still needed to detect and remove different types of noise associated with drivers' data, especially noises caused by body movement (Eilebrecht et al. 2012; Rodrigues et al. 2015).

Another challenge in data quality is correct annotation of recorded data. Recorded signals are commonly annotated by using self-reporting methods, which is entirely subjective, and different drivers may have different psychological responses to the same stressful driving situation. Thus, developing an objective metric to measure driver stress levels can help to address this issue (Deguchi et al. 2006; Eilebrecht et al. 2012; Sharma and Gedeon 2012).

4.6.2 Dataset. Using publicly available datasets has several benefits, such as facilitating a fair comparison of all experiments and reducing the workload of researchers. However, the number of available public datasets in this domain is limited due to ethics and privacy issues. 
Table 5. The Most Common and Important Classification Techniques Used for Different Driver Stress Levels

\begin{tabular}{|c|c|c|c|c|c|}
\hline $\begin{array}{c}\text { Stress } \\
\text { Level/Class }\end{array}$ & Classifier & Data & Performance & Compared with & Reference \\
\hline \multirow{3}{*}{2} & SVM & ECG and EMG & $\begin{array}{l}\text { Accuracy: } 100 \% \\
\quad(10 \text {-fold), } \\
\text { classification rate: } \\
100 \% \text {, sensitivity: } \\
100 \% \text {, specificity: } \\
\quad 100 \%\end{array}$ & $\begin{array}{l}\text { KNN: accuracy: } 100 \%(10 \text {-fold), } \\
\text { classification rate: } 81.26 \% \\
\text { sensitivity: } 62.13 \% \\
\text { specificity: } 88.93 \%\end{array}$ & $\begin{array}{l}\text { Soman et al. } \\
\qquad(2014)\end{array}$ \\
\hline & $\mathrm{KNN}$ & ECG & $\begin{array}{l}\text { Recognition rate: } \\
97.78 \% \\
\text { (5-fold) }\end{array}$ & - & $\begin{array}{l}\text { Wang et al. } \\
\qquad(2013)\end{array}$ \\
\hline & $\mathrm{BN}$ & $\begin{array}{c}\text { ECG, EDA, RSP, } \\
\text { vehicle dynamic data }\end{array}$ & Accuracy: $96 \%$ & - & $\begin{array}{l}\text { Rigas et al. } \\
\text { (2012) }\end{array}$ \\
\hline \multirow{3}{*}{3} & NMC & $\begin{array}{c}\text { ECG, EDA, RSP, } \\
\text { vehicle dynamic data }\end{array}$ & Accuracy: $90 \%$ & $\begin{array}{c}\mathrm{KNN} \text { (no accuracy is reported) } \\
\text { Multilayer perceptron (no accuracy is } \\
\text { reported) }\end{array}$ & $\begin{array}{l}\text { Lanatà et al. } \\
\qquad(2015)\end{array}$ \\
\hline & LRNN & $\begin{array}{l}\text { PPG, EDA, derived } \\
\text { features for } \\
\text { HRV and RSP }\end{array}$ & $\begin{array}{l}\text { Specificity: } 94.92 \% \\
\text { Sensitivity: } 88.83 \% \\
\text { Precision: } 89.23 \% \\
\text { Desirability } \\
\text { measure: } \\
43.11769\end{array}$ & $\begin{array}{c}\text { Feed-forward distributed time-delay } \\
\text { neural network (desirability measure: } \\
23.75342 \text { ), } \\
\text { single-layer perceptron neural } \\
\text { network } \\
\text { (desirability measure: } 8.56352 \text { ), } \\
\text { multilayer perceptron neural network } \\
\text { (desirability measure: } 39.85285 \text { ), } \\
\text { cascade-forward back-propagation } \\
\text { neural network } \\
\text { (desirability measure: } 29.86937), \\
\text { Elman back-propagation neural } \\
\text { network } \\
\text { (desirability measure: } 40.46697), \\
\text { nonlinear autoregressive networks } \\
\text { with exogenous inputs neural } \\
\text { network (desirability measure: } \\
23.96057)\end{array}$ & $\begin{array}{l}\text { Singh et al. } \\
\quad(2013)\end{array}$ \\
\hline & LDA & $\begin{array}{l}\text { ECG, EMG, EDA, } \\
\text { RSP }\end{array}$ & Accuracy: $97.4 \%$ & - & $\begin{array}{l}\text { Healey and } \\
\text { Picard (2005) }\end{array}$ \\
\hline \multirow[t]{3}{*}{4} & $\begin{array}{l}\text { Hybrid decision } \\
\text { tree- tree } \\
\text { augmented NBC }\end{array}$ & $\begin{array}{l}\text { ECG, EDA, RSP, } \\
\text { facial EMG }\end{array}$ & Accuracy: $71.9 \%$ & $\begin{array}{c}\text { Tree-augmented NBC (accuracy: } \\
70.6 \% \text { ), NBC (accuracy: 61.4\%), C4.5 } \\
\text { regression tree classifier (accuracy: } \\
70.1 \% \text { ), KNN (accuracy: 65.5\%), SVM } \\
\text { (accuracy: } 69.3 \% \text { ), ANFIS (accuracy: } \\
68.1 \% \text { ) }\end{array}$ & $\begin{array}{l}\text { Katsis et al. } \\
\qquad(2011)\end{array}$ \\
\hline & SVM & $\begin{array}{l}\text { ECG, EMG, EDA, } \\
\text { RSP }\end{array}$ & Accuracy: $79.3 \%$ & ANFIS (accuracy: 76.7\%) & $\begin{array}{l}\text { Katsis et al. } \\
\quad(2008)\end{array}$ \\
\hline & KNN & ECG, EDA, RSP & Accuracy: $88.6 \%$ & - & $\begin{array}{c}\text { Healey and } \\
\text { Picard }(2000)\end{array}$ \\
\hline
\end{tabular}

The only public driver physiological dataset in this domain was collected by Healey (2000). The database contains a collection of different physiological data (e.g., ECG, EMG, EDA, and RSP) from 17 drivers who were driving around 1 hour or more on Boston city streets and highways. Another dataset includes survey data from a study that assessed drivers' perceived stress in different driving situations, such as driving on various roads, in traffic jams, and in weather-related scenarios (Hill and Boyle 2007). The survey collected information related to the users' requirements from an advanced traveler system ( $\mathrm{Ng}$ et al. 1995). The data was collected through a mail-back survey that was distributed in 1994. 
4.6.3 Modality Fusion. Integrating modalities is an essential approach to obtain a more accurate detection model where unimodal data cannot represent a full understanding of driver stress levels, especially in real-world driving situations. Although modality fusion can help to build a more efficient and accurate model, the main challenge is in integrating the modalities (sensor, feature, score, or decision). Among the different fusion techniques, feature-level fusion has been used in most of the reviewed studies. Although fusion in the feature level has been shown to have an acceptable performance, there are still a range of subissues associated with it, such as asynchrony between the modalities, selecting the optimal window size for different modalities (Rigas et al. 2008), and increasing computational cost by increasing number of features (Rigas et al. 2011). For other fusion techniques, more research is needed to investigate their roles in building a more accurate and reliable driver stress detection system.

4.6.4 Data Analysis. Another challenging issue that affects the performance of a real-time driver stress recognition system is high dimensionality of data. This issue is addressed by using feature-based methods, especially feature selection and reduction. These methods remove the redundant and irrelevant data to improve the performance of the system. Applying and evaluating dimensionality reduction techniques to enhance the automatic detection of driver stress-level performance is an open research challenge that needs to be investigated further. Recently, evolutionary computation algorithms, particularly particle swarm optimization and differential evolution, have been shown to be effective as feature selection methods in affective detection (Nakisa et al. 2017). In addition, the literature suggests it is worth exploring new features and investigating new feature extraction analyses that have greater correlation with driver stress levels. For example, nonlinear analysis of HRV signals (which is in the early stage of use) was shown to have more correlation to stress levels of drivers compared with the frequency domain analysis of HRV signals (Munla et al. 2015).

4.6.5. System Acceptability. Another important issue is the acceptability of such a system by car drivers in terms of the method of collecting the data. A system may be considered acceptable when it can record data in an unobtrusive and noninvasive manner. The data related to driver physical responses and context can be easily tracked and measured in an acceptable way, but the correlation between these types of data and driver stress levels has been investigated much less than physiological signals. Further studies are therefore required to investigate the correlation between these modalities and drivers' stress.

Although physiological signal data has been researched more thoroughly, the body contact sensors that record physiological responses may not be practical for continuous stress monitoring of drivers.

Using body contact sensors is an obtrusive method that can restrict drivers' movements and increase their awareness of being monitored. Using contactless methods to collect biosignals is one proposed strategy to overcome this issue. Some studies monitored body temperature through different drivers' face regions by using a thermal camera to monitor their distraction and stress (Wesley et al. 2010). Wartzek et al. (2011) used a contactless ECG to detect heart rate in different driving situations, especially driving on highways. In some studies (Baek et al. 2009; Hernandez et al. 2014), different biosignals (PPG, ECG, EDA, and RSP activity) were recorded through sensors attached to the driver's seat, seatbelt, and vehicle steering wheel. These sensors provided a convenient data collection method without the driver needing to wear any body contact sensors.

4.6.6 Driving Scenario. It has been proved that drivers' stress responses obtained from simulated and actual driving scenarios are different (Baek et al. 2009). Drivers' tension levels in simulated driving scenarios are low compared to their tension levels in real-time scenarios, since the 
risk of crash is zero (Lee et al. 2007). Therefore, different driver stress levels can be observed better from real-world driving experiments. However, recording stress levels of drivers in real-time environments using intrusive physiological sensors may affect driving safety and cause vehicle crashes. For safety and ethical reasons, it is therefore very hard to evaluate drivers' stress levels in real-time driving situations (Zheng et al. 2015). Today's advanced driving simulators have the advantage of well-designed visual, rotation, and sound systems that provide a highly realistic sensation that is close to a real driving feeling (Yamaguchi et al. 2009). These simulators provide an efficient and reliable way to study driver stress responses by using driving scenarios that are repeatable (Fisher et al. 2011).

\section{CONCLUSION}

Stress is considered to be a main reason for vehicle crashes. Prolonged exposure to different driving stressors may also cause health problems in professional drivers. Due to the ever-increasing fatality rate and economic losses related to driver stress, an in-vehicle system for early detection of driver stress levels is essential to increase drivers' safety. This system should continuously detect drivers' stress levels, predict dangerous driving situations, and, at an early stage of detecting an increase in stress, alert the driver proactively. The findings from several studies in the domain of smart cars have shown that such a system can not only increase driving safety but also cause positive impacts on drivers' driving patterns and behaviors. In addition to improved safety, such a system may have an additional benefit of preventing harmful long-term consequences of stress in professional drivers, and could lead to a more efficient economy, environment (optimizing fuel consumption, greenhouse gas emissions, etc.), and society (social and psychological response) (Flach et al. 2011; Silva et al. 2014).

In this review, a general framework of a multimodal driver stress detection system was presented. This framework involves two main parts: data collection and data analysis. Data collection includes different modalities, such as psychological, physiological, physical, and contextual data, which are used to measure driver stress. We have reviewed the most recent works related to these modalities and discussed their limitations. Data analysis includes computational techniques, such as preprocessing, feature generation, feature selection, feature reduction, and machine-learning techniques, which were comprehensively discussed. A summary of the reviewed literature that used different modalities and machine-learning methods to build a driver stress recognition model can be found in Table 6 .

Among the different modalities, physiological responses have been used widely in the domain of driver stress detection, but there are still some challenges related to this modality that need to be addressed. Generally, physiological changes due to stress are subject dependent and vary from subject to subject. Therefore, it is difficult to make a general model that suits all drivers, and a system would need to be able to adapt itself to each individual's physiology. Another challenge is that body contact sensors for physiological monitoring are not practical for a continuous driver stress-level detection system, since they are too obtrusive for real-world driving situations. Physical and contextual data can be collected using less intrusive and more practical methods suitable for real-life situations. As physical and contextual data are also potentially correlated to stress levels of drivers, future studies should target these data more in building an automatic driver stress detection system.

It has been shown that the different modalities can complement each other to create a more robust driver stress detection model; therefore, considering multimodal approaches to build the optimal system is essential in this domain. One of the major issues is in combining or integrating the modalities in the fusion levels (sensor, feature, score, or decision). Feature-level fusion has been widely used to evaluate drivers' stress levels, but there is also good potential to investigate 


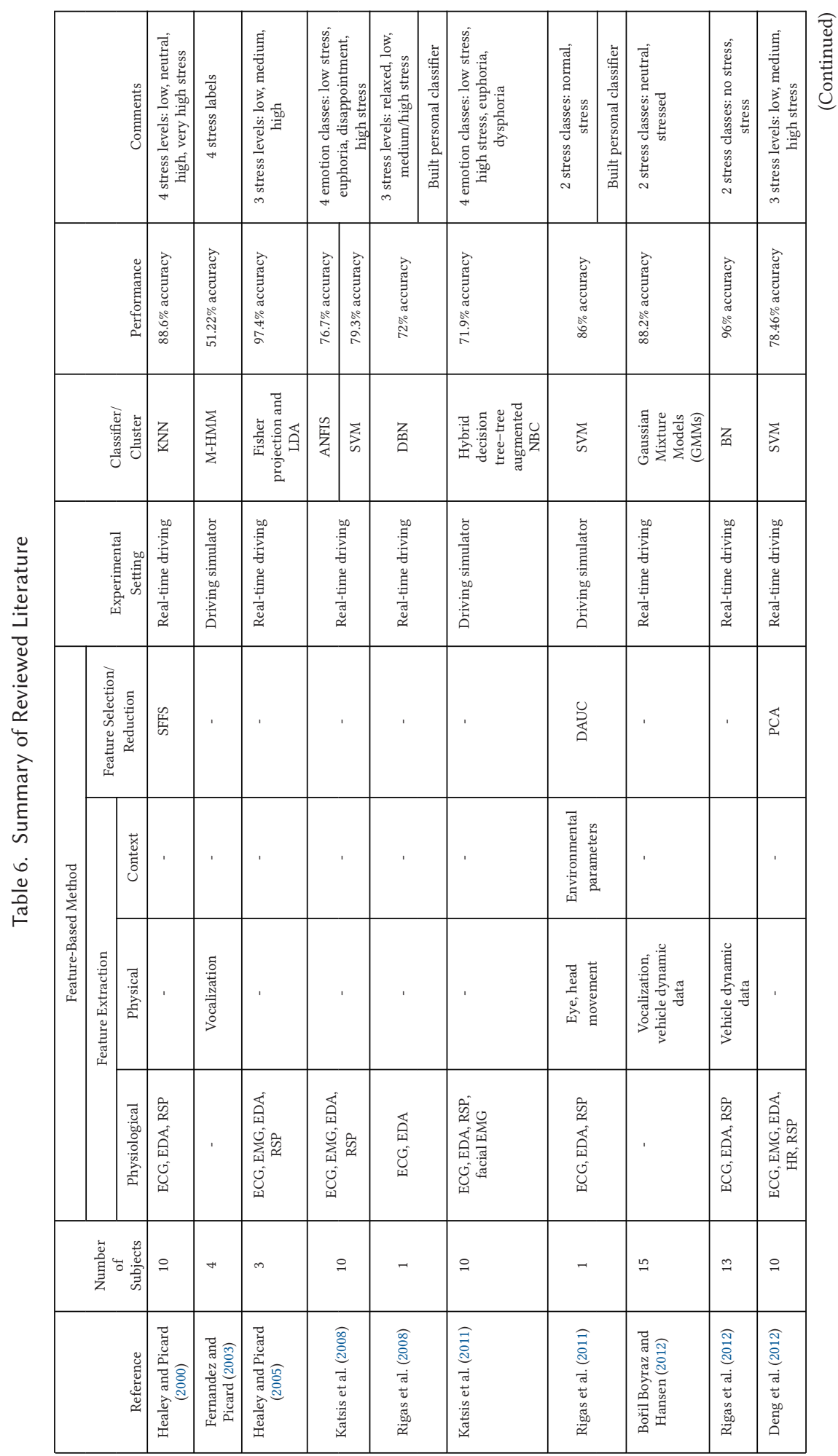




\begin{tabular}{|c|c|c|c|c|c|c|c|c|c|c|c|}
\hline & $\begin{array}{l}\text { औँ } \\
\text { 音 } \\
\text { ठ }\end{array}$ & 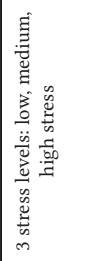 & 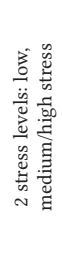 & 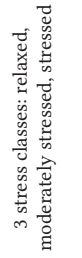 & 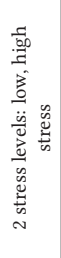 & 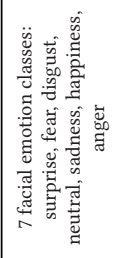 & 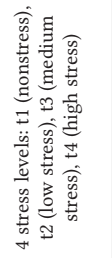 & 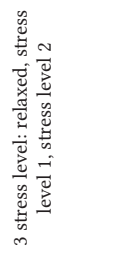 & 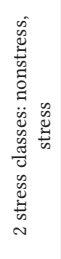 & 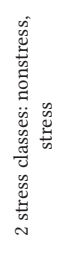 & 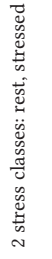 \\
\hline & $\begin{array}{l}\text { : } \\
\text { : } \\
\text { : } \\
\text { : }\end{array}$ & 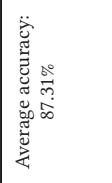 & 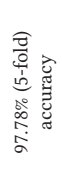 & 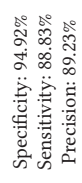 & 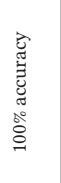 & 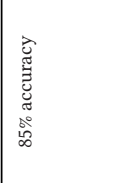 & 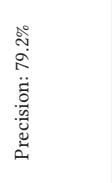 & 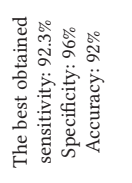 & 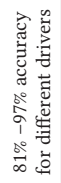 & 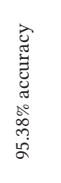 & 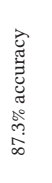 \\
\hline & 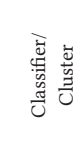 & 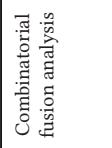 & 总 & 总 & 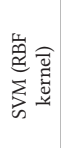 & $\sum_{i}$ & 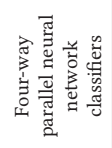 & $\sum_{\Sigma}^{U}$ & 苞 & 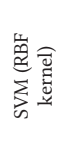 & 兑 \\
\hline & 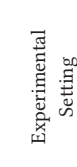 & 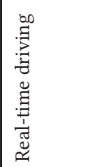 & 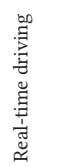 & 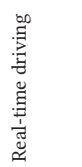 & 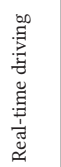 & 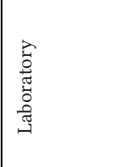 & 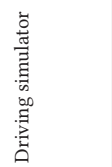 & 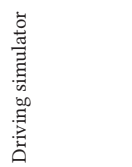 & 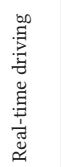 & 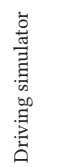 & 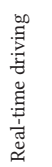 \\
\hline \multirow{4}{*}{ 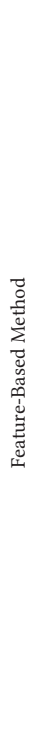 } & 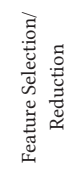 & 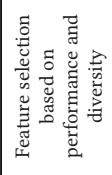 & 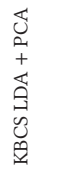 & 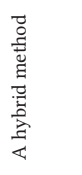 & ' & ' & ' & 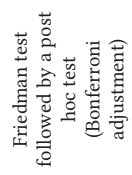 & , & 虫 & 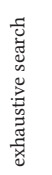 \\
\hline & ठ & ' & ' & ' & ' & ' & ' & ' & ' & ' & ' \\
\hline & 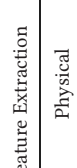 & ' & , & ' & ' & 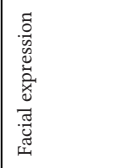 & ' & 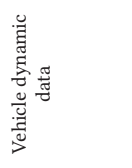 & , & 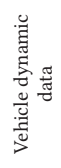 & ' \\
\hline & 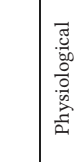 & 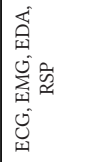 & ن & 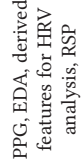 & 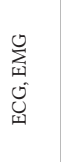 & ' & $\begin{array}{l}\text { 苗 } \\
\text { 宫 }\end{array}$ & 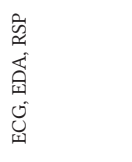 & 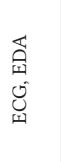 & 产 & 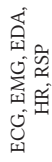 \\
\hline & 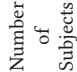 & $\stackrel{\circ}{\circ}$ & $\stackrel{\circ}{=}$ & $\stackrel{\partial}{-}$ & $\exists$ & Fे & $\ddot{m}$ & 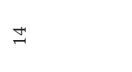 & 0 & $\stackrel{\infty}{N}$ & $a$ \\
\hline & 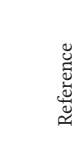 & 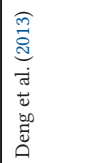 & 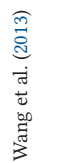 & 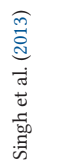 & 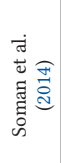 & 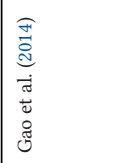 & 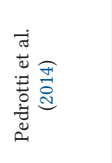 & 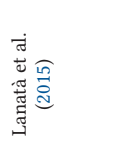 & 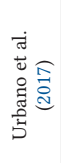 & 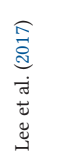 & 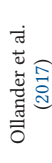 \\
\hline
\end{tabular}


score-level and decision-level fusion in future studies and improve the performance of the model. Thus, integration of different modalities to create a more robust and unobtrusive early detection system for driver stress levels, which can reduce current stress-related driving problems, is suggested for future work. Applying effective data collection and analysis methods in order to address methodological issues to build such a system is also recommended for further research.

\section{ACKNOWLEDGMENTS}

The authors would like to gratefully thank Ms. Margaret Ann Duffield for her help and copy editing services provided by Golden Orb Creative, NSW, Australia.

\section{REFERENCES}

A. Akbas. 2011. Evaluation of the physiological data indicating the dynamic stress level of drivers. Scientific Research and Essays 6, 2 (2011), 430-439.

E. Alba, J. García-Nieto, L. Jourdan, and E.-G. Talbi. 2007. Gene selection in cancer classification using PSO/SVM and GA/SVM hybrid algorithms. In 2007 IEEE Congress on Evolutionary Computation. IEEE, 284-290. Retrieved from http://ieeexplore.ieee.org/xpls/abs_all.jsp?arnumber $=4424483$.

A. Alberdi, A. Aztiria, and A. Basarab. 2016. Towards an automatic early stress recognition system for office environments based on multimodal measurements: A review. Fournal of Biomedical Informatics 59 (2016), 49-75. Retrieved from https://doi.org/10.1016/j.jbi.2015.11.007.

M. T. Allen, C. M. Stoney, J. F. Owens, and K. A. Matthews. 1993. Hemodynamic adjustments to laboratory stress: The influence of gender and personality. Psychosomatic Medicine 55, 6 (1993), 505-517.

N. S. Altman. 1992. An introduction to kernel and nearest-neighbor nonparametric regression. American Statistician 46, 3 (1992), 175-185

P. Angkititrakul, M. Petracca, A. Sathyanarayana, and J. H. Hansen. 2007. UTDrive: Driver behavior and speech interactive systems for in-vehicle environments. In 2007 IEEE Intelligent Vehicles Symposium. IEEE, 566-569. Retrieved from http://ieeexplore.ieee.org/xpls/abs_all.jsp?arnumber $=4290175$.

C. Avcı, A. Akbaş, and Y. Yüksel. (n.d.). Evaluation of statistical metrics by using physiological data to identify the stress level of drivers. Retrieved from http://www.ipcbee.com/vol78/022-ICECB2014-S0007.pdf.

M. Aydede and P. Robbins. 2009. The Cambridge Handbook of Situated Cognition. Cambridge University Press, New York. Retrieved from http://www.upbo.org/catalogue/catalogue.asp?isbn=9780521612869\&ss=exc.

A. D. Baddeley. 1972. Selective attention and performance in dangerous environments. British fournal of Psychology 63, 4 (1972), 537-546.

H. J. Baek, H. B. Lee, J. S. Kim, J. M. Choi, K. K. Kim, and K. S. Park. 2009. Nonintrusive biological signal monitoring in a car to evaluate a driver's stress and health state. Telemedicine and E-Health 15, 2 (2009), 182-189.

R. M. Baevskii, A. P. Berseneva, E. Y. Bersenev, and A. K. Eshmanova. 2009. Use of principles of prenosological diagnosis for assessing the functional state of the body under stress conditions as exemplified by bus drivers. Human Physiology 35, 1 (2009) 34-42. Retrieved from https://doi.org/10.1134/S0362119709010071

A. Baum. 1997. Cambridge Handbook of Psychology, Health and Medicine. Cambridge University Press. Retrieved from https://books.google.com.au/books?hl=en\&lr=\&id=zVh30FrAuDsC\&oi=fnd\&pg=PR13\&dq=Cambridge+ Handbook+of+Psychology, +Health+and+Medicine\&ots=Ik9NoDuNur\&sig=8WdUp0xNImLcvvCvyKvnD98wido.

V. Beanland, M. Fitzharris, K. L. Young, and M. G. Lenné. 2013. Driver inattention and driver distraction in serious casualty crashes: Data from the Australian national crash in-depth study. Accident Analysis \& Prevention 54 (2013), 99-107. Retrieved from https://doi.org/10.1016/j.aap.2012.12.043.

D. J. Beirness. 1993. Do we really drive as we live? The role of personality factors in road crashes. Alcohol, Drugs \& Driving 9, 3 (1993), 129-143. Retrieved from http://psycnet.apa.org/psycinfo/1994-47349-001.

L. M. Bergasa, J. M. Buenaposada, J. Nuevo, P. Jimenez, and L. Baumela. 2008. Analysing driver's attention level using computer vision. In 2008 11th International IEEE Conference on Intelligent Transportation Systems. IEEE, 1149-1154. Retrieved from http://ieeexplore.ieee.org/xpls/abs_all.jsp?arnumber=4732544.

W. Berger, I. Figueira, A. M. Maurat, E. P. Bucassio, I. Vieira, S. R. Jardim, and M. V. Mendlowicz. 2007. Partial and full PTSD in Brazilian ambulance workers: prevalence and impact on health and on quality of life. fournal of Traumatic Stress 20 , 4 (2007), 637-642.

J. Blasco, T. M. Chen, J. Tapiador, and P. Peris-Lopez. 2016. A survey of wearable biometric recognition systems. ACM Computing Surveys (CSUR) 49, 3 (2016), 43.

L. Blincoe, T. R. Miller, E. Zaloshnja, and B. A. Lawrence. 2015. The Economic and Societal Impact of Motor Vehicle Crashes, 2010 (Revised). Retrieved from https://trid.trb.org/view.aspx?id=1311862. 
H. Bořil, P. Boyraz, and J. H. L. Hansen. 2012. Towards multimodal driver's stress detection. In Digital Signal Processing for In-Vehicle Systems and Safety, J. H. L. Hansen, P. Boyraz, K. Takeda, and H. Abut (Eds.), Springer New York, 3-19. Retrieved from https://doi.org/10.1007/978-1-4419-9607-7_1.

H. Bořil, S. Omid Sadjadi, T. Kleinschmidt, and J. H. Hansen. 2010. Analysis and detection of cognitive load and frustration in drivers' speech. In 2010 11th Annual Conference of the International Speech Communication Association (INTERSPEECH'10). 502-505.

R. Boyer and A. Brunet. 1996. Prevalence of post-traumatic stress disorder in bus drivers. Sante Mentale Au Quebec 21, 1 (1996), 189-208.

L. N. Boyle, J. Tippin, A. Paul, and M. Rizzo. 2008. Driver performance in the moments surrounding a microsleep. Transportation Research Part F: Traffic Psychology and Behaviour 11, 2 (2008), 126-136.

J. J. Braithwaite, D. G. Watson, R. Jones, and M. Rowe. 2013. A guide for analysing electrodermal activity (EDA) \& skin conductance responses (SCRs) for psychological experiments. Psychophysiology 49 (2013), 1017-1034.

A. J. Camm, M. Malik, J. T. Bigger, G. Breithardt, S. Cerutti, R. J. Cohen, P. Coumel, E. L. Fallen, H. L. Kennedy, R. E. Kleiger, F. Lombardi, A. Malliani, A. J. Moss, J. N. Rottman, G. Schmidt, P. J. Schwartz, and D. H. Singer. 1996. Heart rate variability. Standards of measurement, physiological interpretation, and clinical use. European Heart fournal 17, 3 (1996), 354-381.

V. Cantin, M. Lavallière, M. Simoneau, and N. Teasdale. 2009. Mental workload when driving in a simulator: Effects of age and driving complexity. Accident Analysis \& Prevention 41, 4 (2009), 763-771.

C. Carter and R. Graham. 2000. Experimental comparison of manual and voice controls for the operation of in-vehicle systems. Proceedings of the Human Factors and Ergonomics Society Annual Meeting 44, 20 (2000), 3-286. Retrieved from https://doi.org/10.1177/154193120004402016.

G. Chandrashekar and F. Sahin. 2014. A survey on feature selection methods. Computers \& Electrical Engineering 40, 1 (2014), 16-28.

F. J. Chang and Y.-T. Chang. 2006. Adaptive neuro-fuzzy inference system for prediction of water level in reservoir. Advances in Water Resources 29, 1 (2006), 1-10. Retrieved from https://doi.org/10.1016/j.advwatres.2005.04.015.

F. Chen, N. Ruiz, E. Choi, J. Epps, M. A. Khawaja, R. Taib, and Y. Wang. 2012. Multimodal behavior and interaction as indicators of cognitive load. ACM Transactions on Interactive Intelligent Systems (TiiS) 2, 4 (2012), 22.

M. Chennaoui, C. Bougard, C. Drogou, C. Langrume, C. Miller, D. Gomez-Merino, and F. Vergnoux. 2016. Stress biomarkers, mood states, and sleep during a major competition: "Success" and "failure" athlete's profile of high-level swimmers. Frontiers in Physiology 7 (2016), 94. Retrieved from https://doi.org/10.3389/fphys.2016.00094.

N. Copsey, L. Drupsteen, J. Kampen, L. van Kuijt-Evers, E. Schmitz-Felten, and M. Verjans. 2010. A review of accidents and injuries to road transport drivers. Retrieved from http://www.narcis.nl/publication/RecordID/oai:tudelft.nl:uuid\% 3Ae9a20676-31e7-48c1-9b3c-439bb62a99dd.

J. L. Deffenbacher, D. M. Deffenbacher, R. S. Lynch, and T. L. Richards. 2003. Anger, aggression, and risky behavior: A comparison of high and low anger drivers. Behaviour Research and Therapy 41, 6 (2003), 701-718.

M. Deguchi, J. Wakasugi, T. Ikegami, S. Nanba, and M. Yamaguchi. 2006. Evaluation of driver stress using motor-vehicle driving simulator. IEEf Transactions on Sensors and Micromachines 126 (2006), 438-444. Retrieved from https://doi.org/ 10.1541/ieejsmas.126.438.

Y. Deng, Z. Wu, C. H. Chu, and T. Yang. 2012. Evaluating feature selection for stress identification. In 2012 IEEE 13th International Conference on Information Reuse and Integration (IRI'12), 584-591. Retrieved from https://doi.org/10.1109/ IRI.2012.6303062.

Z. Deng Yong Wu, C.-H. Chu, Q. Zhang, and D. F. Hsu. 2013. Sensor feature selection and combination for stress identification using combinatorial fusion. International fournal of Advanced Robotic Systems 10 (2013), 306.

B. Eilebrecht, S. Wolter, J. Lem, H. Lindner, R. Vogt, M. Walter, and S. Leonhardt. 2012. The relevance of HRV parameters for driver workload detection in real world driving. In 2012 Computing in Cardiology (CinC'12). IEEE, 409-412. Retrieved from http://ieeexplore.ieee.org/xpls/abs_all.jsp?arnumber=6420417.

M. Elgendi. 2012. On the analysis of fingertip photoplethysmogram signals. Current Cardiology Reviews 8, 1 (2012), 14-25. Retrieved from https://doi.org/10.2174/157340312801215782.

H. J. Eysenck and S. B. G. Eysenck. 1975. Manual of the Eysenck Personality Questionnaire (funior and Adult). Hodder and Stoughton.

G. W. Evans and S. Carrère. 1991. Traffic congestion, perceived control, and psychophysiological stress among urban bus drivers. Journal of Applied Psychology 76, 5 (1991), 658.

R. Fernandez and R. W. Picard. 2003. Modeling drivers' speech under stress. Speech Communication 40, 1 (2003), 145-159.

D. L. Fisher, M. Rizzo, J. Caird, and J. D. Lee. 2011. Handbook of Driving Simulation for Engineering, Medicine, and Psychology. CRC Press. Retrieved from https://books.google.com.au/books?hl=en\&lr=\&id=lnTA8sT8gkEC\&oi=fnd\&pg=PP1\&dq= Fisher, +D.L.\%3B+Rizzo, +M.\%3B+Caird,+J.K.\%3B+Lee, +J.D.+Handbook+of+Driving+Simulation+for+Engineering, + Medicine, + and +Psychology\%3B+CRC+Press:+New+York,+NY,+USA+2011.\&ots=GUuo_Y9DrR\&sig=kk1SCSwmITzk Zuj2T8Rh8yE_usY. 
T. Flach, N. Mishra, L. Pedrosa, C. Riesz, and R. Govindan. 2011. CarMA. In Proceedings of the 9th ACM Conference on Embedded Networked Sensor Systems (SenSys'11), 135.

C. Frasson, P. O. Brosseau, and T. H. D. Tran. 2014. Virtual environment for monitoring emotional behaviour in driving. In Intelligent Tutoring Systems. Springer, 75-83. Retrieved from http://link.springer.com/chapter/10.1007/ 978-3-319-07221-0_10.

N. Friedman, D. Geiger, and M. Goldszmidt. 1997. Bayesian network classifiers. Machine Learning 29, 2-3 (1997), 131-163.

A. Furnham and J. Saipe. 1993. Personality correlates of convicted drivers. Personality and Individual Differences 14, 2 (1993), 329-336.

C. Fyfe. 2005. Artificial neural networks. In Do Smart Adaptive Systems Exist? B. Gabrys, K. Leiviskä, \& J. Strackeljan (Eds.). Springer, Berlin, 57-79. Retrieved from https://doi.org/10.1007/3-540-32374-0_4.

H. Gao, A. Yuce, and J.-P. Thiran. 2014. Detecting emotional stress from facial expressions for driving safety. In 2014 IEEE International Conference on Image Processing (ICIP'14). IEEE, 5961-5965. Retrieved from http://ieeexplore.iee.org/xpls/ abs_all.jsp?arnumber $=7026203$.

Z. Ghahramani. 2001. An introduction to hidden Markov models and bayesian networks. International fournal of Pattern Recognition and Artificial Intelligence 15, 1 (2001), 9-42.

E. Gulian, G. Matthews, A. I. Glendon, D. R. Davies, and L. M. Debney. 1989. Dimensions of driver stress. Ergonomics 32, 6 (1989), 585-602.

H. Gunes and M. Pantic. 2010. Automatic measurement of affect in dimensional and continuous spaces: Why, what, and how? In Proceedings of the 7th International Conference on Methods and Techniques in Behavioral Research. ACM, 12. Retrieved from http://dl.acm.org/citation.cfm?id=1931356.

D. J. Hand, H. Mannila, and P. Smyth. 2001. Principles of Data Mining. MIT Press. Retrieved from https://books.google. com.au/books?hl=en\&lr=\&id=SdZ-bhVhZGYC\&oi=fnd\&pg=PR17\&dq=Hand, + D. + J., + Mannila,$+H .,+\% 26+$ Smyth,$+P$. $+(2001)+$ +Principles + of + data + mining. + Cambridge,+ Mass.: + MIT + Press.\&ots $=y x S 3$ tmuosX\&sig=xUrE1Qn3Pu3D1_sd Fgvsq6QAvo.

I. Hanzlíková. 2005. Professional drivers: The sources of occupational stress. In Ponencia Presentada en el Seminario Young Researchers Seminar. Retrieved from http://www.ectri.org/YRS05/Papiers/Session-4/hanzlikova.pdf.

L. R. Hartley, P. K. Arnold, G. Smythe, and J. Hansen. 1994. Indicators of fatigue in truck drivers. Applied Ergonomics 25, 3 (1994), 143-156.

J. Healey, J. Seger, and R. Picard. 1999. Quantifying driver stress: developing a system for collecting and processing biometric signals in natural situations. Biomedical Sciences Instrumentation 35 (1999), 193-198.

J. A. Healey. 2000. Wearable and Automotive Systems for Affect Recognition from Physiology. Massachusetts Institute of Technology.

J. Healey and R. Picard. 2000. SmartCar: Detecting driver stress. In Proceedings of the 15th International Conference on Pattern Recognition, 2000. IEEE, 4, 218-221. Retrieved from http://ieeexplore.ieee.org/xpls/abs_all.jsp?arnumber=902898.

J. A. Healey and R. W. Picard. 2005. Detecting stress during real-world driving tasks using physiological sensors. IEEE Transactions on Intelligent Transportation Systems 6, 2 (2005), 156-166.

D. D. Heikoop, J. C. F. de Winter, B. van Arem, and N. A. Stanton. 2017. Effects of platooning on signal-detection performance, workload, and stress: A driving simulator study. Applied Ergonomics 60 (Supplement C), 116-127. Retrieved from https://doi.org/10.1016/j.apergo.2016.10.016.

D. Hemenway and S. J. Solnick. 1993. Fuzzy dice, dream cars, and indecent gestures: Correlates of driver behavior? Accident Analysis \& Prevention 25, 2 (1993), 161-170.

D. A. Hennessy, D. L. Wiesenthal, and P. M. Kohn. 2000. The influence of traffic congestion, daily hassles, and trait stress susceptibility on state driver stress: An interactive perspective1. Journal of Applied Biobehavioral Research 5, 2 (2000), $162-179$.

J. Hernandez, D. McDuff, X. Benavides, J. Amores, P. Maes, and R. Picard. 2014. Autoemotive: Bringing empathy to the driving experience to manage stress. In Proceedings of the 2014 Companion Publication on Designing Interactive Systems. ACM, 53-56. Retrieved from http://dl.acm.org/citation.cfm?id=2602780.

J. D. Hill and L. N. Boyle. 2007. Driver stress as influenced by driving maneuvers and roadway conditions. Transportation Research Part F: Traffic Psychology and Behaviour 10, 3 (2007), 177-186. Retrieved from https://doi.org/10.1016/j.trf.2006. 09.002 .

R. Horlings, D. Datcu, and L. J. Rothkrantz. 2008. Emotion recognition using brain activity. In Proceedings of the 9th International Conference on Computer Systems and Technologies and Workshop for PhD Students in Computing. ACM, 6. Retrieved from http://dl.acm.org/citation.cfm?id=1500888.

C.-W. Hsu, C.-C. Chang, and C.-J. Lin. 2003. A practical guide to support vector classification. Retrieved from https://www.cs.sfu.ca/people/Faculty/teaching/726/spring11/svmguide.pdf.

W. C. Huang, C. M. Lai, B. Luo, C. K. Tsai, M. H. Chih, C. W. Lai, and H. T. Lin. 2006. Intelligent model-based OPC. In Optical Microlithography XIX, 6154:615436. International Society for Optics and Photonics. Retrieved from https:/ doi.org/10.1117/12.657792. 
J.-S. R. Jang. 1991. Fuzzy modeling using generalized neural networks and Kalman filter algorithm. In AAAI, 91, 762-767). Retrieved from http://www.aaai.org/Library/AAAI/1991/aaai91-119.php.

G. H. John and P. Langley. 1995. Estimating continuous distributions in bayesian classifiers. In Proceedings of the 11th Conference on Uncertainty in Artificial Intelligence. Morgan Kaufmann Publishers, 338-345. Retrieved from http://dl acm.org/citation.cfm?id=2074196.

L. M. John, R. Flin, and K. Mearns. 2006. Bus driver well-being review: 50 years of research. Transportation Research Part F: Traffic Psychology and Behaviour 9, 2 (2006), 89-114.

C. Kappeler-Setz, F. Gravenhorst, J. Schumm, B. Arnrich, and G. Tröster. 2013. Towards long term monitoring of electrodermal activity in daily life. Personal and Ubiquitous Computing 17, 2 (2013), 261-271.

D. Katsis Christos, N. Katertsidis, G. Ganiatsas, and D. Fotiadis. 2008. Toward emotion recognition in car-racing drivers: A biosignal processing approach. IEEE Transactions on Systems, Man and Cybernetics, Part A: Systems and Humans 38, 3 (2008), 502-512.

D. Katsis Christos, Y. Goletsis, G. Rigas, and D. I. Fotiadis. 2011. A wearable system for the affective monitoring of car racing drivers during simulated conditions. Transportation Research Part C: Emerging Technologies 19, 3 (2011), 541-551.

D. Katsis Christos, G. Rigas, Y. Goletsis, and D. I. Fotiadis. 2015. Emotion recognition in car industry. In Emotion Recognition, A. Konar \& A. Chakraborty (Eds.). John Wiley \& Sons, 515-544. Retrieved from http://onlinelibrary.wiley.com/doi/10. 1002/9781118910566.ch20/summary.

S. S. Kia, S. Noroozi, B. Carse, J. Vinney, and M. Rabbani. 2006. Prediction of failure in pin-joints using hybrid adaptive neuro-fuzzy approach. In 2006 IEEE International Conference on Fuzzy Systems, 671-677. Retrieved from https://doi.org/ 10.1109/FUZZY.2006.1681783.

K. Kira and L. A. Rendell. 1992. A practical approach to feature selection. In Proceedings of the 9th International Workshop on Machine Learning, 249-256. Retrieved from https://books.google.com.au/books?hl=en\&lr=\&id=jEejBQAAQBAJ\&oi= fnd\&pg=PA249\&dq=A+practical+approach+to+feature+selection.\&ots=EQ7yliGi_u\&sig=O749h_BKYv3-niXM1XJV92 kFDlY.

M. Kudo and J. Sklansky. 2000. Comparison of algorithms that select features for pattern classifiers. Pattern Recognition 33, 1 (2000), 25-41.

A. Lanatà, G. Valenza, A. Greco, C. Gentili, R. Bartolozzi, F. Bucchi, and E. P. Scilingo. 2015. How the autonomic nervous system and driving style change with incremental stressing conditions during simulated driving. IEEE Transactions on Intelligent Transportation Systems 16, 3 (2015), 1505-1517.

D. S. Lee, T. W. Chong, and B. G. Lee. 2017. Stress events detection of driver by wearable glove system. IEEE Sensors fournal 17, 1 (2017), 194-204. Retrieved from https://doi.org/10.1109/JSEN.2016.2625323.

J. S. Lee, H. B. Kim, Y. S. Kim, H. J. Baek, M. S. Ryu, and K. S. Park. 2007. The relationship between HRV parameters and stressful driving situation in the real road. In 2007 6th International Special Topic Conference on Information Technology Applications in Biomedicine. IEEE, 198-200. Retrieved from http://ieeexplore.ieee.org/xpls/abs_all.jsp?arnumber=4407380.

M. G. Lenné, T. J. Triggs, and J. R. Redman. 1997. Time of day variations in driving performance. Accident Analysis \& Prevention, 29, 4 (1997), 431-437.

N. Li and C. Busso. 2013. Analysis of facial features of drivers under cognitive and visual distractions. In 2013 IEEE International Conference on Multimedia and Expo (ICME'13). IEEE, 1-6. Retrieved from http://ieeexplore.ieee.org/xpls/abs_all. jsp?arnumber $=6607575$.

N. Li, J. J. Jain, and C. Busso. 2013. Modeling of driver behavior in real world scenarios using multiple noninvasive sensors. IEEE Transactions on Multimedia 15, 5 (2013), 1213-1225.

Q. Liu and J. Wang. 2008. A one-layer recurrent neural network for convex programming. In 2008 IEEE International foint Conference on Neural Networks (IEEE World Congress on Computational Intelligence). IEEE, 83-90. Retrieved from http:// ieeexplore.ieee.org/xpls/abs_all.jsp?arnumber $=4633771$.

S.-H. Liu, C.-T. Lin, and W.-H. Chao. 2004. The short-time fractal scaling of heart rate variability to estimate the mental stress of driver. In 2004 IEEE International Conference on Networking, Sensing and Control. IEEE, 829-833. Retrieved from http://ieeexplore.ieee.org/xpls/abs_all.jsp?arnumber=1297054.

European Working Conditions Survey. 2005. European Foundation for the Improvement of Living and Working Conditions (Eurofound), data extracted by Prevent for OSH Data Collection 2007. https://www.eurofound.europa.eu/surveys/ european-working-conditions-surveys/fourth-european-working-conditions-survey-2005.

U. Lundberg, R. Kadefors, B. Melin, G. Palmerud, P. Hassmén, M. Engström, and I. E. Dohns. 1994. Psychophysiological stress and EMG activity of the trapezius muscle. International fournal of Behavioral Medicine 1, 4 (1994), 354-370.

G. Marquart, C. Cabrall, and J. de Winter. 2015. Review of eye-related measures of drivers' mental workload. Procedia Manufacturing 3 (2015), 2854-2861.

G. Matthews, L. Dorn, and A. I. Glendon. 1991. Personality correlates of driver stress. Personality and Individual Differences 12, 6 (1991), 535-549.

G. Matthews, L. Dorn, T. W. Hoyes, D. R. Davies, A. I. Glendon, and R. G. Taylor. 1998. Driver stress and performance on a driving simulator. Human Factors: The fournal of the Human Factors and Ergonomics Society 40, 1 (1998), 136-149. 
L. Medsker and L. C. Jain. 1999. Recurrent Neural Networks: Design and Applications. CRC Press. Retrieved from https:// books.google.com.au/books?hl=en\&lr=\&id=ME1SAkN0PyMC\&oi=fnd\&pg=PA1\&dq=Layer+recurrent+neural+ networks+(LRNN)\&ots=7aAsaO8UUr\&sig=_jlvudyDBKBGfilYSUzy-gmca1I.

E. E. Miller and L. N. Boyle. 2013. Variations in road conditions on driver stress insights from an on-road study. In Proceedings of the Human Factors and Ergonomics Society Annual Meeting. SAGE Publications, 57, 1864-1868. Retrieved from http://pro.sagepub.com/content/57/1/1864.short.

H. Min and W. Fangfang. 2010. Filter-wrapper hybrid method on feature selection. In 2010 2nd WRI Global Congress on Intelligent Systems (GCIS’10). IEEE, 3, 98-101.

A. Monroe. 2009. Understanding and managing peripheral neuropathy. BETA: Bulletin of Experimental Treatments for AIDS: A Publication of the San Francisco AIDS Foundation 22, 2 (2009), 27-35.

N. Montano, T. G. Ruscone, A. Porta, F. Lombardi, M. Pagani, and A. Malliani. 1994. Power spectrum analysis of heart rate variability to assess the changes in sympathovagal balance during graded orthostatic tilt. Circulation 90, 4 (1994), $1826-1831$.

N. Munla, M. Khalil, A. Shahin, and A. Mourad. 2015. Driver stress level detection using HRV analysis. In 2015 International Conference on Advances in Biomedical Engineering (ICABME'15), 61-64. Retrieved from https://doi.org/10.1109/ICABME. 2015.7323251.

K. P. Murphy. 2002. Dynamic bayesian networks. Probabilistic Graphical Models, M. Fordan, 7. Retrieved from http:// webdocs.cs.ualberta.ca/ greiner/C-366/366-SLIDES/dbn-murphy.pdf.

B. Nakisa, M. N. Rastgoo, D. Tjondronegoro, and V. Chandran. 2017. Evolutionary computation algorithms for feature selection of EEG-based emotion recognition using mobile sensors. Expert Systems with Applications 93 (2017), 143-155. Retrieved from https://doi.org/10.1016/j.eswa.2017.09.062.

L. Ng, W. Barfield, and F. Mannering. 1995. A survey-based methodology to determine information requirements for advanced traveler information systems. Transportation Research Part C: Emerging Technologies 3, 2 (1995), 113-127.

R. W. Novaco, D. Stokols, and L. Milanesi. 1990. Objective and subjective dimensions of travel impedance as determinants of commuting stress. American fournal of Community Psychology 18, 2 (1990), 231-257.

S. Ollander, C. Godin, S. Charbonnier, and A. Campagne. 2017. Feature and sensor selection for detection of driver stress. Presented at the 3rd International Conference on Physiological Computing Systems, 115-122. Retrieved from http:// www.scitepress.org/DigitalLibrary/Link.aspx?doi=10.5220/0005973901150122.

M. H. Parry. 1968. Aggression on the Road. JSTOR.

M. Paschero, G. Del Vescovo, L. Benucci, A. Rizzi, M. Santello, G. Fabbri, and F. F. Mascioli. 2012. A real time classifier for emotion and stress recognition in a vehicle driver. In 2012 IEEE International Symposium on Industrial Electronics (ISIE'12). IEEE, 1690-1695. Retrieved from http://ieeexplore.ieee.org/xpls/abs_all.jsp?arnumber=6237345.

M. Pedrotti, M. A. Mirzaei, A. Tedesco, J.-R. Chardonnet, F. Mérienne, S. Benedetto, and T. Baccino. 2014. Automatic stress classification with pupil diameter analysis. International fournal of Human-Computer Interaction 30, 3 (2014), 220-236.

F. N. Platt. 1970. Heart rate measurements of drivers with the highway systems research car. Fournal of the Medical Association of Georgia, 59 (HS-010 737). Retrieved from https://trid.trb.org/view.aspx?id=115573.

D. Price. 2010. How to read an electrocardiogram (ECG). Part 1: Basic principles of the ECG. The normal ECG. Southern Sudan Medical fournal 3, 2 (2010), 26-28.

G. Rigas, C. D. Katsis, P. Bougia, and D. I. Fotiadis. 2008. A reasoning-based framework for car driver's stress prediction. In 2008 16th Mediterranean Conference on Control and Automation. IEEE, 627-632. Retrieved from http://ieeexplore.ieee. org/xpls/abs_all.jsp?arnumber=4602162.

G. Rigas, Y. Goletsis, P. Bougia, and D. I. Fotiadis. 2011. Towards driver's state recognition on real driving conditions. International Journal of Vehicular Technology (2011), 617210. Retrieved from http://www.hindawi.com/journals/ijvt/2011/ 617210/abs/.

G. Rigas, Y. Goletsis, and D. Fotiadis. 2012. Real-time driver's stress event detection. IEEE Transactions on Intelligent Transportation Systems 13, 1 (2012), 221-234.

M. Rimini-Doering, D. Manstetten, T. Altmueller, U. Ladstaetter, and M. Mahler. 2001. Monitoring driver drowsiness and stress in a driving simulator. In Driving Assessment. Retrieved from http://drivingassessment.uiowa.edu/DA2001/10_ Rimini_Doering_Maria.pdf.

J. G. P. Rodrigues, M. Kaiseler, A. Aguiar, J. P. S. Cunha, and J. Barros, 2015. A mobile sensing approach to stress detection and memory activation for public bus drivers. IEEE Transactions on Intelligent Transportation Systems 16, 6 (2015), 32943303. Retrieved from https://doi.org/10.1109/TITS.2015.2445314.

L. J. Rothkrantz, P. Wiggers, J.-W. A. van Wees, and R. J. van Vark. 2004. Voice stress analysis. In Text, Speech and Dialogue. Springer, 449-456. Retrieved from http://link.springer.com/chapter/10.1007/978-3-540-30120-2_57.

S. R. Safavian and D. Landgrebe. 1990. A survey of decision tree classifier methodology. Retrieved from http://ntrs.nasa. gov/search.jsp?R=19910014493.

A. Sahayadhas, K. Sundaraj, and M. Murugappan. 2012. Detecting driver drowsiness based on sensors: A review. Sensors 12,12 (2012), 16937-16953. 
R. Sahni. 2012. Noninvasive monitoring by photoplethysmography. Clinics in Perinatology 39, 3 (2012), 573-583. Retrieved from https://doi.org/10.1016/j.clp.2012.06.012.

V. A. Sauerzapf. 2012. Road Traffic Crash Fatalities: An Examination of National Fatality Rates and Factors Associated with the Variation in Fatality Rates between Nations with Reference to the World Health Organisation Decade of Action for Road Safety 2011-2020. University of East Anglia. Retrieved from https://ueaeprints.uea.ac.uk/id/eprint/46589.

R. A. Schmidt and T. D. Lee. 2005. Sensory contributions to motor control. Motor Control and Learning: A Behavioural Emphasis 4 (2005), 125-162.

H. Selye. 1956. The stress of life. Retrieved from http://psycnet.apa.org/psycinfo/1957-08247-000.

H. Selye. 1974. Stress without distress. New York, 26-39.

N. Sharma and T. Gedeon. 2012. Objective measures, sensors and computational techniques for stress recognition and classification: A survey. Computer Methods and Programs in Biomedicine 108, 3 (2012), 1287-1301. Retrieved from https:// doi.org/10.1016/j.cmpb.2012.07.003.

F. Silva, C. Analide, C. Gonçalves, and J. Sarmento. 2014. Ubiquitous sensorization for multimodal assessment of driving patterns. In Ambient Intelligence-Software and Applications. Springer, 143-150. Retrieved from http://link.springer.com/ chapter/10.1007/978-3-319-07596-9_16.

F. Simon and C. Corbett. 1996. Road traffic offending, stress, age, and accident history among male and female drivers. Ergonomics 39, 5 (1996), 757-780.

E. Simonson, C. Baker, N. Burns, C. Keiper, O. H. Schmitt, and S. Stackhouse. 1968. Cardiovascular stress (electrocardiographic changes) produced by driving an automobile. American Heart fournal 75, 1 (1968), 125-135.

M. Singh and A. B. Queyam. 2013a. A novel method of stress detection using physiological measurements of automobile drivers. International fournal of Electronics Engineering 5, 2 (2013), 13-20.

M. Singh and A. B. Queyam. 2013b. Correlation between physiological parameters of automobile drivers and traffic conditions. International fournal of Electronics Engineering 5, 2 (2013), 6-12.

R. R. Singh, S. Conjeti, and R. Banerjee. 2013. A comparative evaluation of neural network classifiers for stress level analysis of automotive drivers using physiological signals. Biomedical Signal Processing and Control 8, 6 (2013), 740-754.

K. Soman, V. Alex, and C. Srinivas. 2013. Analysis of physiological signals in response to stress using ECG and respiratory signals of automobile drivers. In 2013 International Multi-Conference on Automation, Computing, Communication, Control and Compressed Sensing (iMac4s'13), 574-579. Retrieved from https://doi.org/10.1109/iMac4s.2013.6526476.

K. Soman, A. Sathiya, and N. Suganthi. 2014. Classification of stress of automobile drivers using radial basis function kernel support vector machine. In 2014 International Conference on Information Communication and Embedded Systems (ICICES'14), 1-5. Retrieved from https://doi.org/10.1109/ICICES.2014.7034000.

L. Sörnmo and P. Laguna. 2006. Electrocardiogram (ECG) signal processing. In Wiley Encyclopedia of Biomedical Engineering. John Wiley \& Sons. Retrieved from http://onlinelibrary.wiley.com/doi/10.1002/9780471740360.ebs1482/abstract.

N. A. Stanton and P. M. Salmon. 2009. Human error taxonomies applied to driving: A generic driver error taxonomy and its implications for intelligent transport systems. Safety Science 47, 2 (2009), 227-237.

N. Sümer. 2003. Personality and behavioral predictors of traffic accidents: Testing a contextual mediated model. Accident Analysis \& Prevention 35, 6 (2003), 949-964.

F. Tango and M. Botta. 2013. Real-time detection system of driver distraction using machine learning. IEEE Transactions on Intelligent Transportation Systems 14, 2 (2013), 894-905.

P. Tchankue, J. Wesson, and D. Vogts. 2013. Using machine learning to predict the driving context whilst driving. In Proceedings of the South African Institute for Computer Scientists and Information Technologists Conference. ACM, 47-55. Retrieved from http://dl.acm.org/citation.cfm?id=2513472.

S. Theodoridis and K. Koutroumbas. 2006. Pattern Recognition. 3rd ed. Academic Press, Orlando, FL.

G. Underwood, P. Chapman, S. Wright, and D. Crundall. 1999. Anger while driving. Transportation Research Part F: Traffic Psychology and Behaviour 2, 1 (1999), 55-68.

M. Urbano, M. Alam, J. Ferreira, J. Fonseca, and P. Simíões. 2017. Cooperative driver stress sensing integration with eCall system for improved road safety. In 17th International Conference on Smart Technologies (IEEE EUROCON'17), 883-888. Retrieved from https://doi.org/10.1109/EUROCON.2017.8011238.

H. Wang and S. Liu. 2016. An effective feature selection approach using the hybrid filter wrapper. International fournal of Hybrid Information Technology 9, 1 (2016), 119-128.

J.-S. Wang, C.-W. Lin, and Y.-T. C. Yang. 2013. A k-nearest-neighbor classifier with heart rate variability feature-based transformation algorithm for driving stress recognition. Neurocomputing 116 (2013), 136-143.

T. Wartzek, B. Eilebrecht, J. Lem, H.-J. Lindner, S. Leonhardt, and M. Walter. 2011. ECG on the road: Robust and unobtrusive estimation of heart rate. IEEE Transactions on Biomedical Engineering 58, 11 (2011), 3112-3120.

A. Wesley, D. Shastri, and I. Pavlidis. 2010. A novel method to monitor driver's distractions. In CHI'10 Extended Abstracts on Human Factors in Computing Systems. ACM, 4273-4278. Retrieved from https://doi.org/10.1145/1753846.1754138. 
H. Wiberg, E. Nilsson, P. Lindén, B. Svanberg, and L. Poom. 2015. Physiological responses related to moderate mental load during car driving in field conditions. Biological Psychology 108 (2015), 115-125. Retrieved from https://doi.org/10.1016/ j.biopsycho.2015.03.017.

J. L. P. Wijsman. 2014. Sensing stress: Stress detection from physiological variables in controlled and uncontrolled conditions. Retrieved from http://eprints.eemcs.utwente.nl/25701/.

D. Yamaguchi, Y. Suda, M. Onuki, T. Oda, H. Ishikawa, and H. Ilgi. 2009. Improvement of realistic sensation on universal driving simulator. In Proceedings of the 16th ITS World Congress, 17.

M. Yamaguchi, J. Wakasugi, and J. Sakakima. 2006. Evaluation of driver stress using biomarker in motor-vehicle driving simulator. In 28th Annual International Conference of the IEEE Engineering in Medicine and Biology Society, 2006 (EMBS'06), 1834-1837. Retrieved from https://doi.org/10.1109/IEMBS.2006.260001.

T. Yamakoshi, K. Yamakoshi, S. Tanaka, M. Nogawa, S. B. Park, M. Shibata, and Y. Hirose. 2008. Feasibility study on driver's stress detection from differential skin temperature measurement. In 200830 th Annual International Conference of the IEEE Engineering in Medicine and Biology Society, 1076-1079. Retrieved from https://doi.org/10.1109/IEMBS.2008.4649346.

T. Yamakoshi, K. Yamakoshi, S. Tanaka, M. Nogawa, M. Shibata, Y. Sawada, and Y. Hirose. 2007. A preliminary study on driver's stress index using a new method based on differential skin temperature measurement. In 2007 29th Annual International Conference of the IEEE Engineering in Medicine and Biology Society, 722-725. Retrieved from https://doi. org/10.1109/IEMBS.2007.4352392.

R. Zheng, S. Yamabe, K. Nakano, and Y. Suda. 2015. Biosignal analysis to assess mental stress in automatic driving of trucks: Palmar perspiration and masseter electromyography. Sensors 15, 3 (2015), 5136-5150.

Received February 2017; revised December 2017; accepted February 2018 